\title{
(4) AEROSPACE

\section{Root Cause Determination of On-orbit Degradation of the VIIRS Rotating Telescope Assembly}

M.R. Ciofalo, P.D. Fuqua, J.D. Barrie, M.J. Meshishnek, C.T. Chu, J.A. Chaney, and R.M. Moision,

The Aerospace Corporation

August 30, 2012 


\section{Suomi National Polar-orbiting Partnership (Suomi NPP)}

- Next-generation Earth-observing research satellite

- Launched 280ct2011

- Sun-synchronous polar orbit

- Approximately 14 orbits per day

- Five payloads

- Advance Technology Microwave Sounder (ATMS)

- Cross-track Infrared Sounder (CrIS)

- Ozone Mapping and Profiler Suite (OMPS)

- Visible Infrared Imaging Radiometer Suite (VIIRS)

- Clouds and the Earth's Radiant Energy System (CERES)
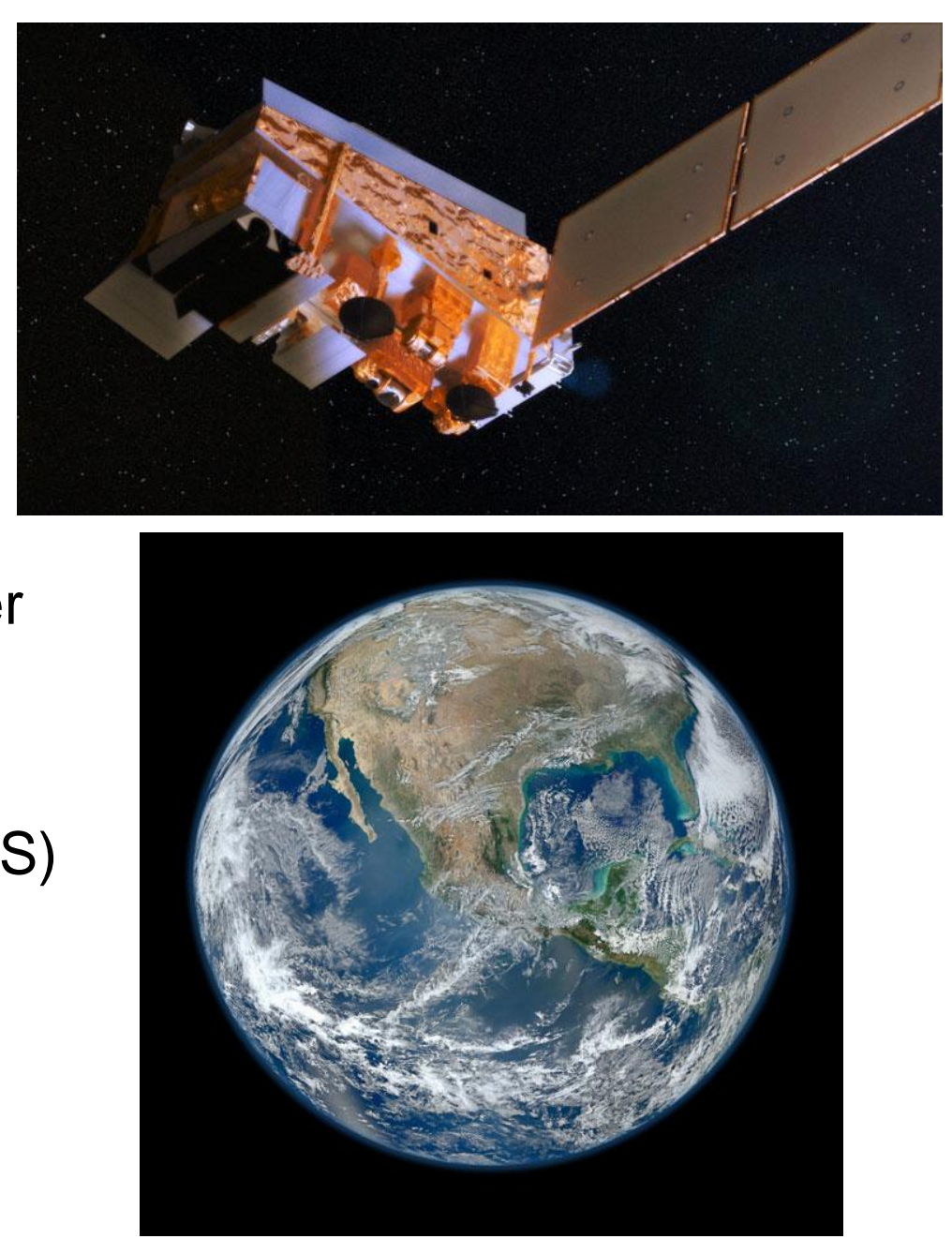

Images courtesy of NASA (http://npp.gsfc.nasa.gov) 


\section{VIIRS}

- Rotating Telescope Assembly (RTA), Fore optics, Aft optics

- 10 silver mirrors

- Dichroics separate beam into:

- Vis/NIR (10 bands)

- Reflective IR (8 bands)

- Thermal IR (4 bands)

- Radiometric calibration required for science missions

- Once per orbit, sunlight illuminates diffuser material

- Intensity recorded by calibration sensor (SDSM)

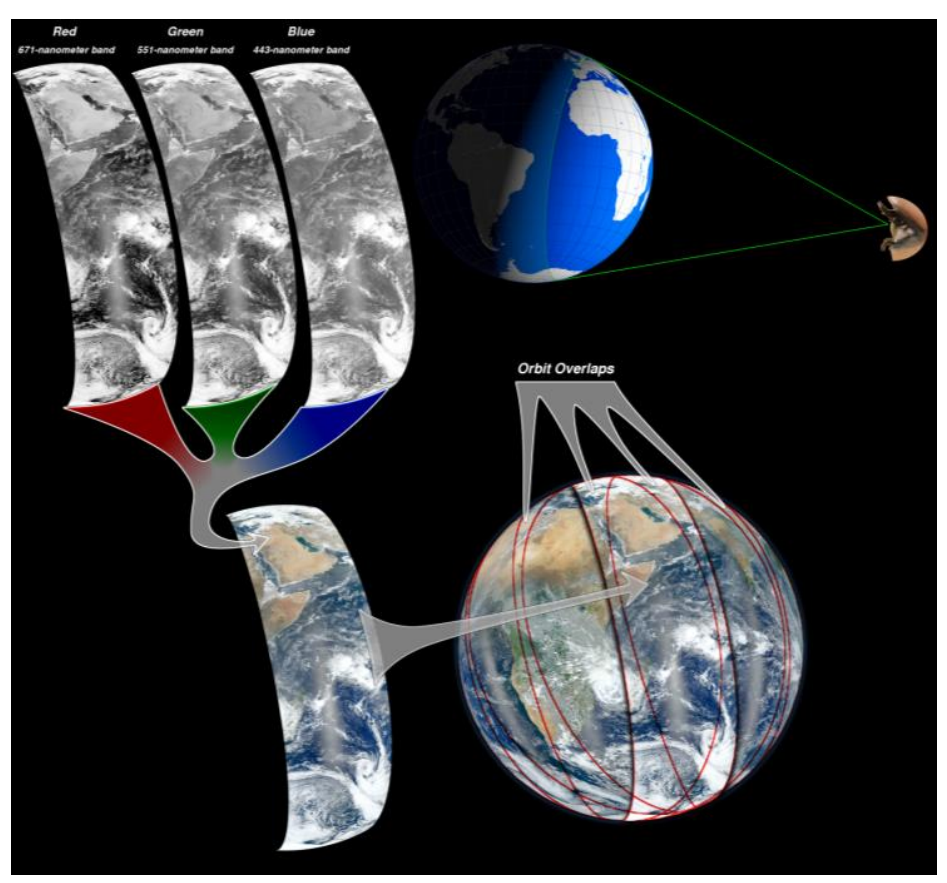

Image courtesy of NASA (http://npp.gsfc.nasa.gov)

- Compared to intensity seen by VIIRS via RTA

- Sensor mass: 252 kg; Coatings: < $5 \mathrm{~g}$ 


\section{VIIRS On-Orbit Degradation}

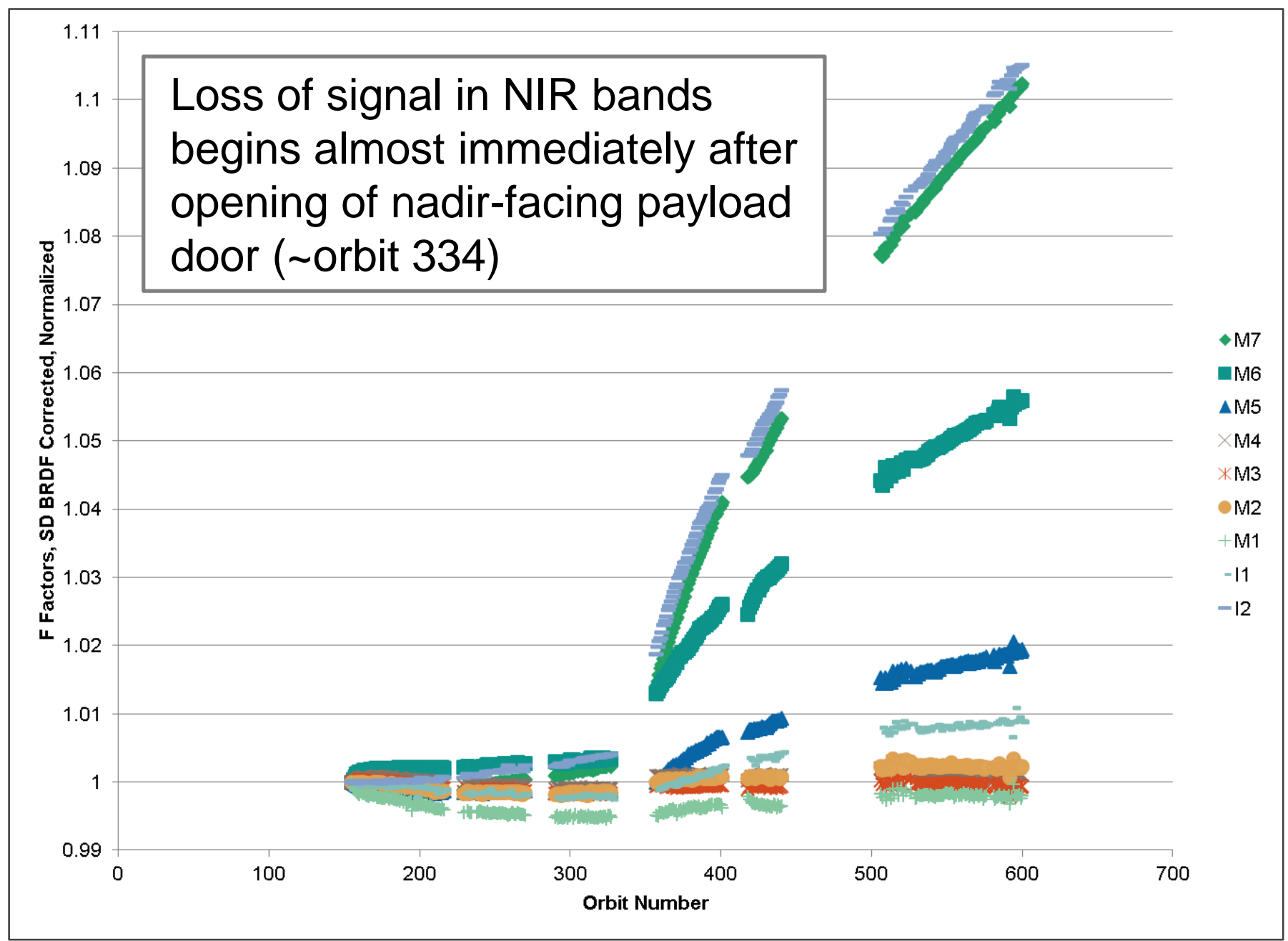

Data courtesy of: Moyer, Rausch, Cardema, Haas, and DeLuccia (13Dec11) 


\section{Root-Cause Investigation of VIIRS Degradation}

- On-orbit data suggests most likely cause is UV-induced degradation of the telescope mirrors

- Mirrors coated in 2004

- Coating has extensive flight heritage

- Root-cause hypotheses proposed:

- Inherent coating defect

- Contamination prior to launch

- Contamination after launch

- The Aerospace Corporation's experience with mirrors focuses investigation onto mechanisms that could induce absorption at the top surface of coatings

- Darkening of dielectric films or deposition of metallic contamination considered much more likely than molecular contamination 


\section{Space Environment Effects (SEE) Testing of Mirror Coatings (2003)}

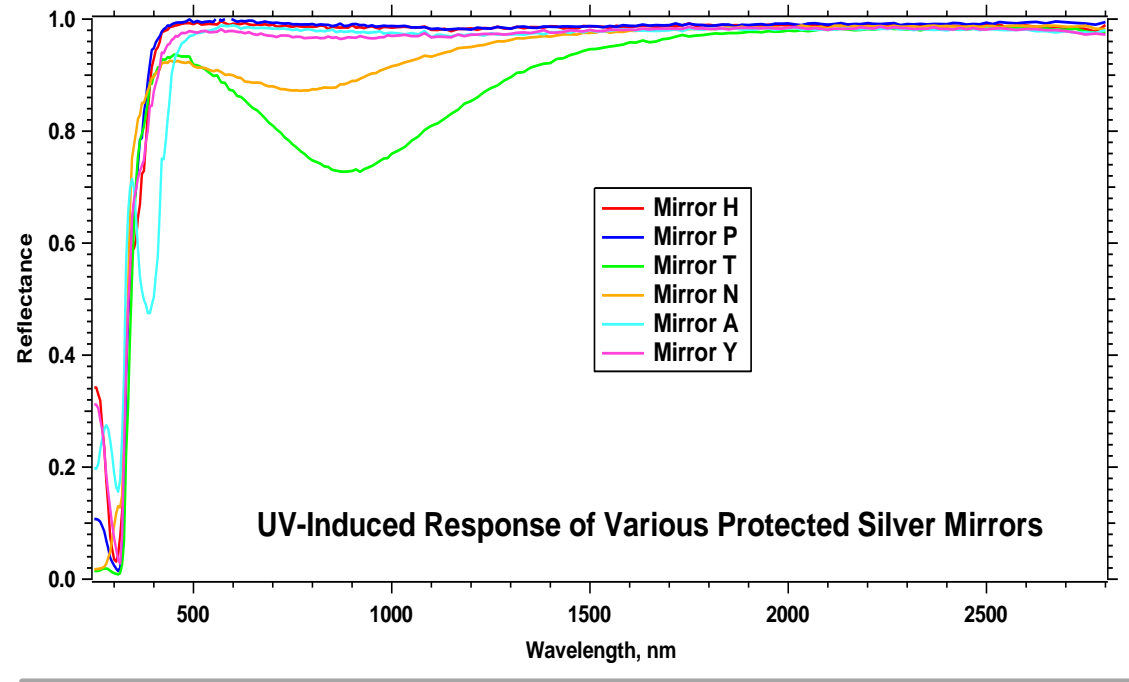

- UV and/or electron radiation can induce absorptions in protected silver mirrors.

- Tests on a variety of mirror types yielded varying results - susceptibility depends upon materials/processes used.

- These results were reported to the VIIRS PO in 2005, but was considered a low risk for their flight-proven coating - contractors did not pursue testing.
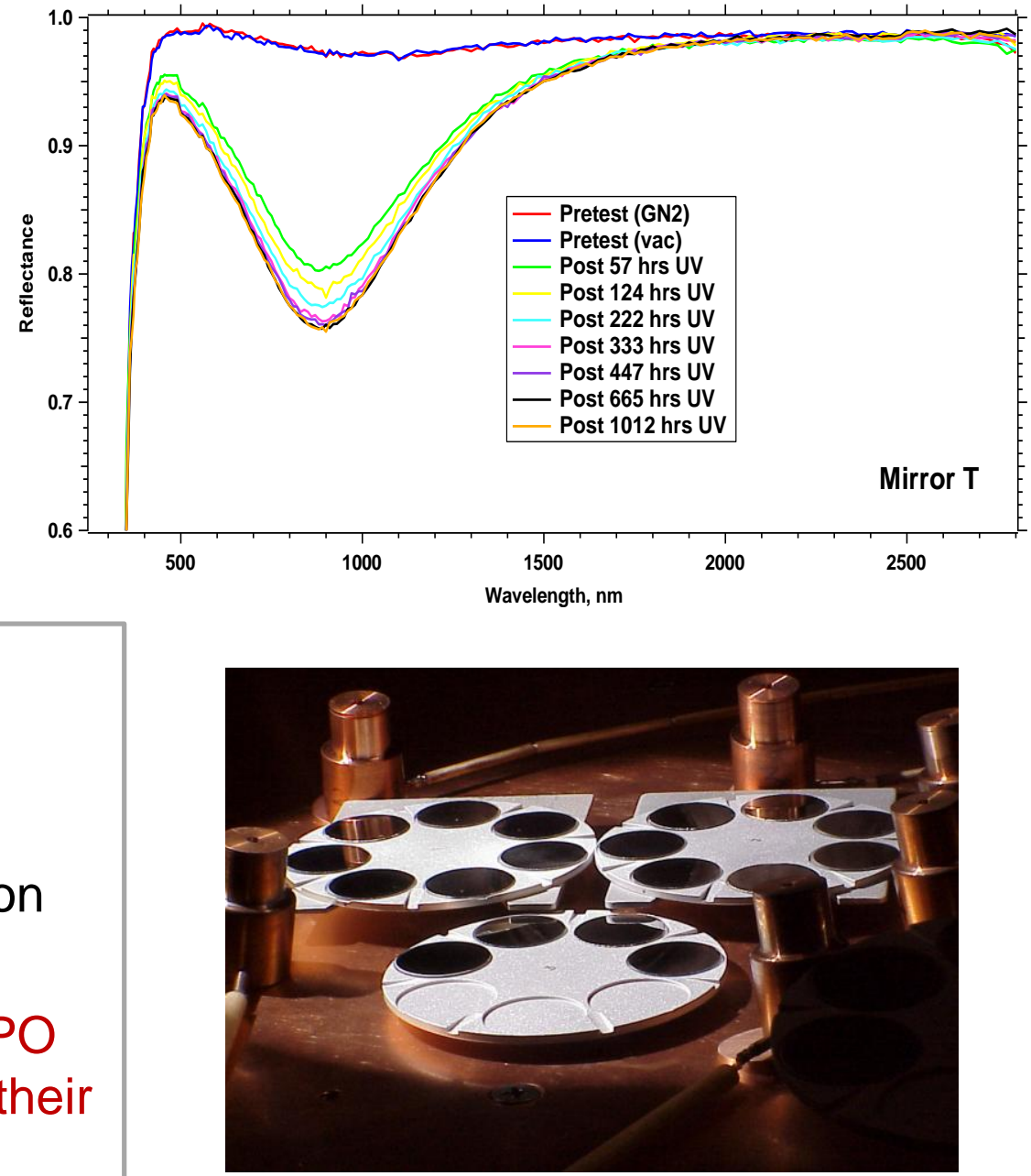

Mirrors under test in SEE chamber 


\section{Root-Cause Investigation of VIIRS Degradation}

- Samples obtained for analysis of surface contaminants

- Witness coupon from the same coating run that produced the VIIRS RTA primary mirror [designated TWM]

- This mirror traveled with VIIRS throughout AI\&T

- Another mirror from the same vendor (different coating run)

- Procured by The Aerospace Corporation (Aerospace) in 2006 as part of a long-term aging study [designated A3-31]

- A CERES mirror (different design) that also traveled with the spacecraft throughout AI\&T

- ToF-SIMS* analysis revealed tungsten oxide on the surface of TWM

- Aerospace's Space Environmental Effects Group prepares one of its facilities for UV-exposure testing of obtained samples

* Time-of-flight Secondary Ion Mass Spectrometry 


\section{R2D2* Space Environmental Effects Exposure Facility}

- Ultra-high-vacuum base-pressure: 2×10-9 Torr

- Broadband UV (200-400nm) Solar Simulator:

- 7-kW Xenon arc lamp

- water-filtered beam reduces IR on samples

- Vacuum UV (115-180 nm) source:

- 150-W Deuterium arc lamp

- 1-100 keV electrons

- In vacuo material characterization

- Spectroreflectometer (250-2800 nm)

- Fiber-optically coupled transmission spectrometer (200-1100 nm)

- FL-IR imaging of samples under test

- Vacuum conditions continuously monitored via Residual Gas Analyzer

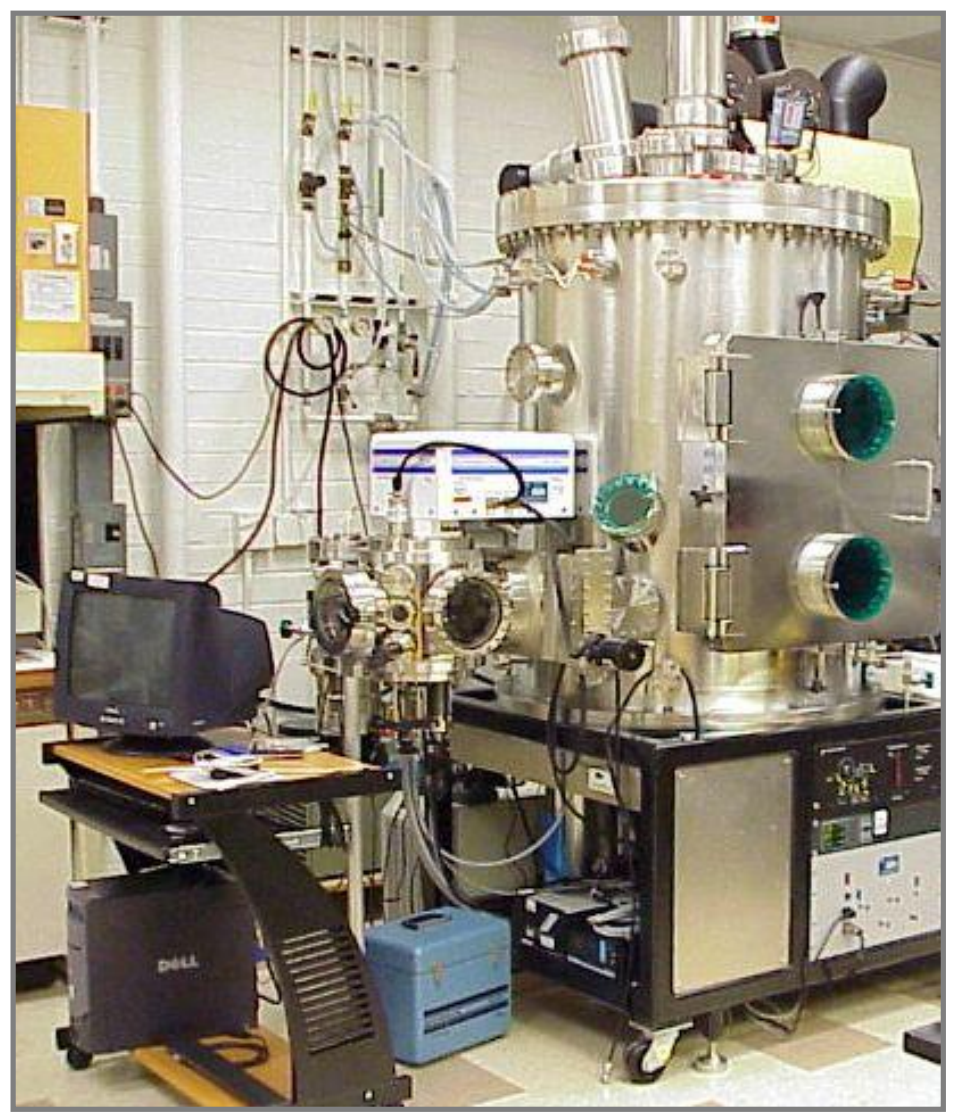

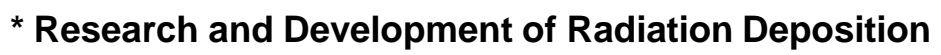




\section{Test Samples}

- Telescope Witness Mirror (TWM)

- A3-31

- CERES mirror

- Control Materials

- 2-mil Rear-Surface-Aluminized Kapton

- 2-mil Rear-Surface-Silverized Teflon (AgFEP)

- Z93-P White Paint

- Contamination Monitors

- Vapor Deposited Aluminum (VDA) Front-Surface Mirror

- 7980 Fused Silica

- Polished Silicon Wafer

\section{Sample matrix exposed to Xe illumination} equivalent to 1-sun intensity. 


\section{Albedo}

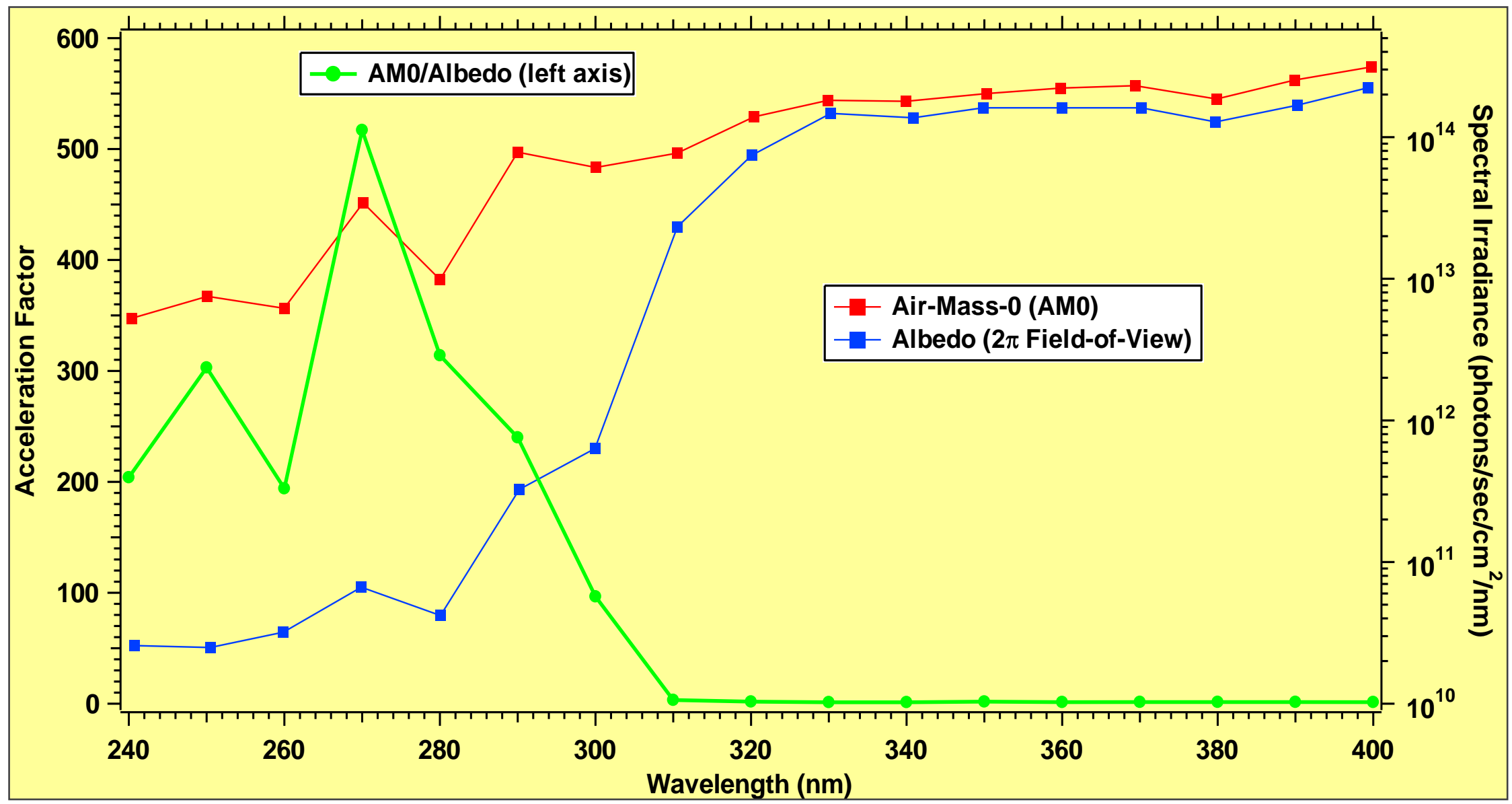

\section{Albedo UV is different from direct solar exposure.}

Albedo data from GOME satellite (https://earth.esa.int) 


\section{Sample Matrix Under Test}

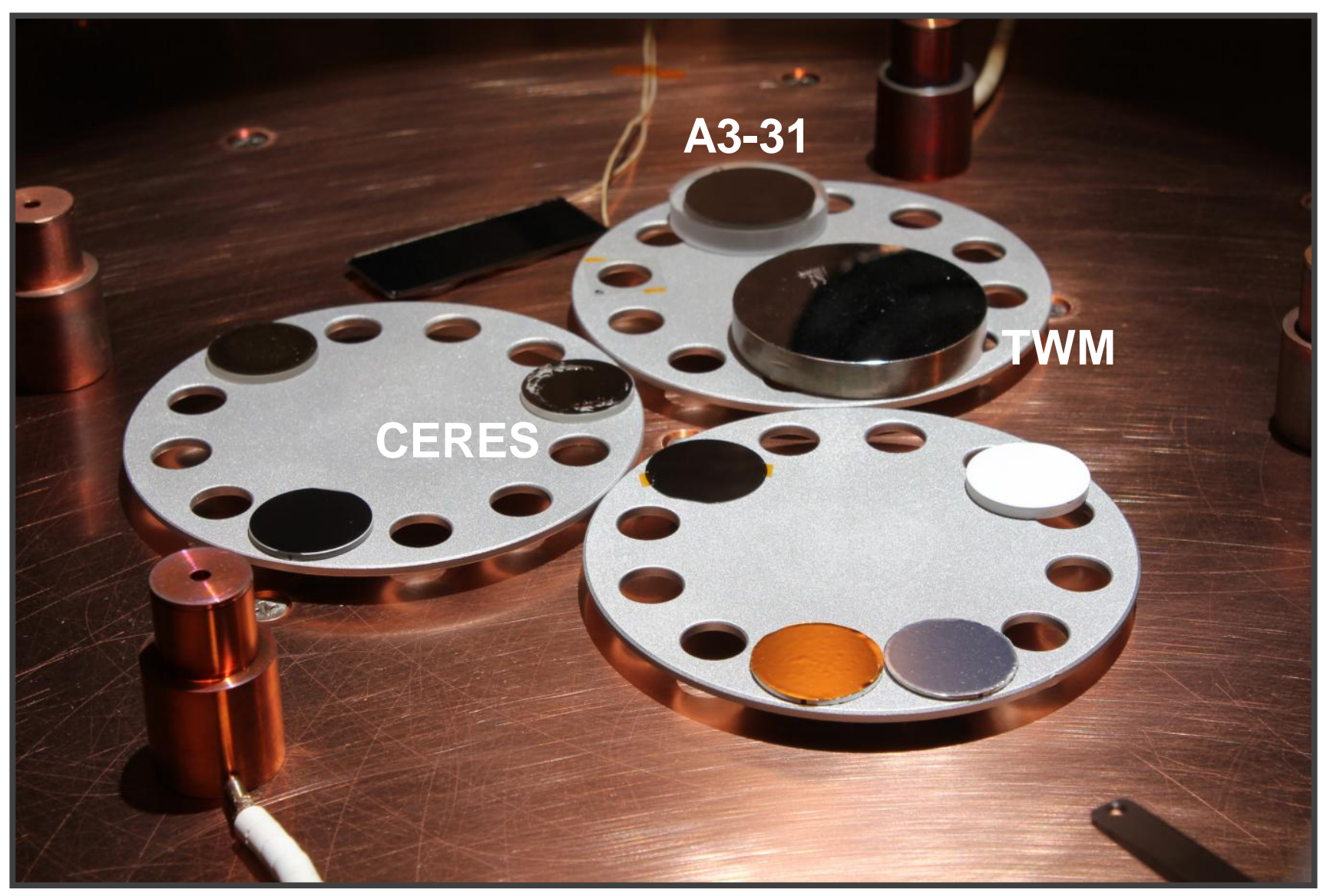

Sample table regulated to $-10^{\circ} \mathrm{C}$ to reduce sample heating. 


\section{TWM measured in 3 places}

$R, C, L=$ right, center, left

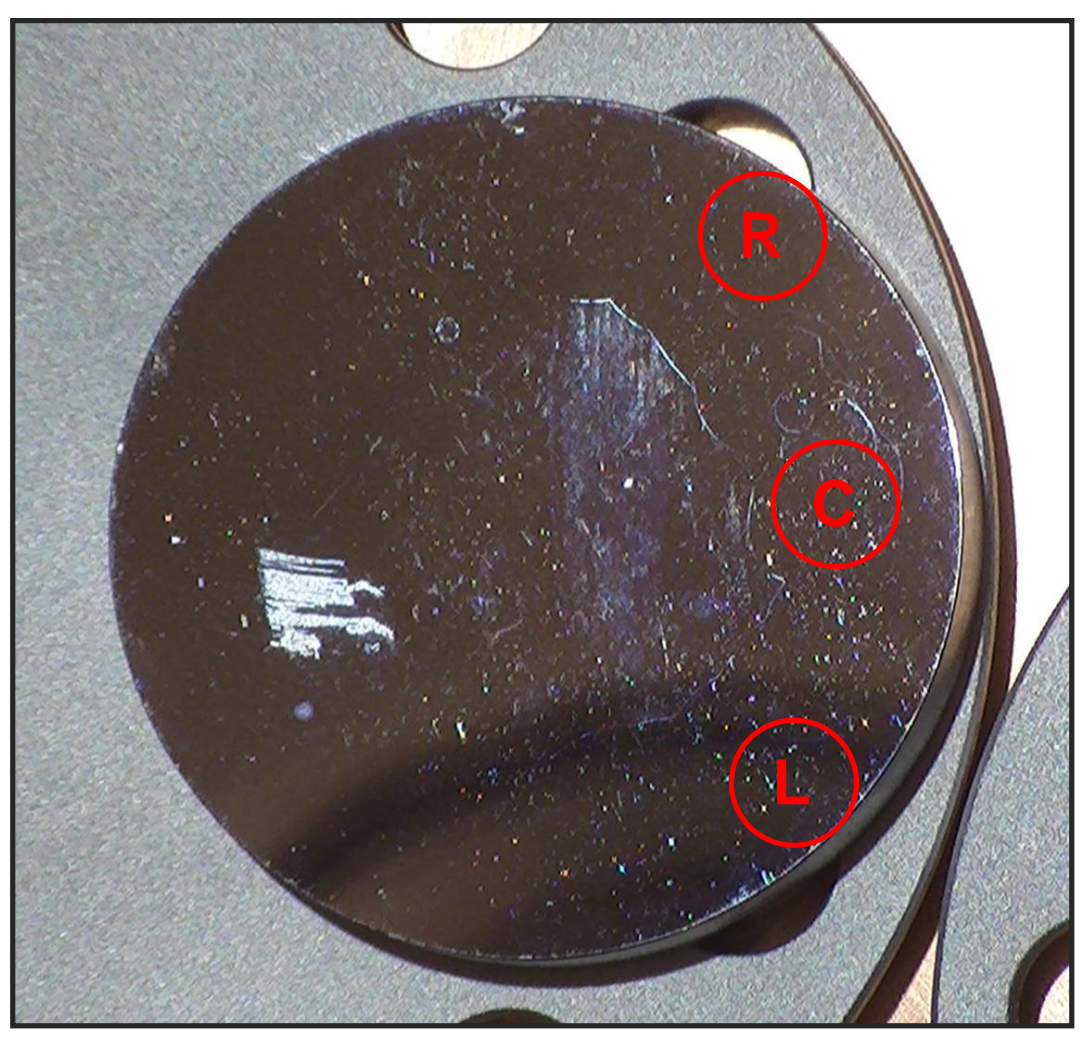




\section{TWM Reflectance}

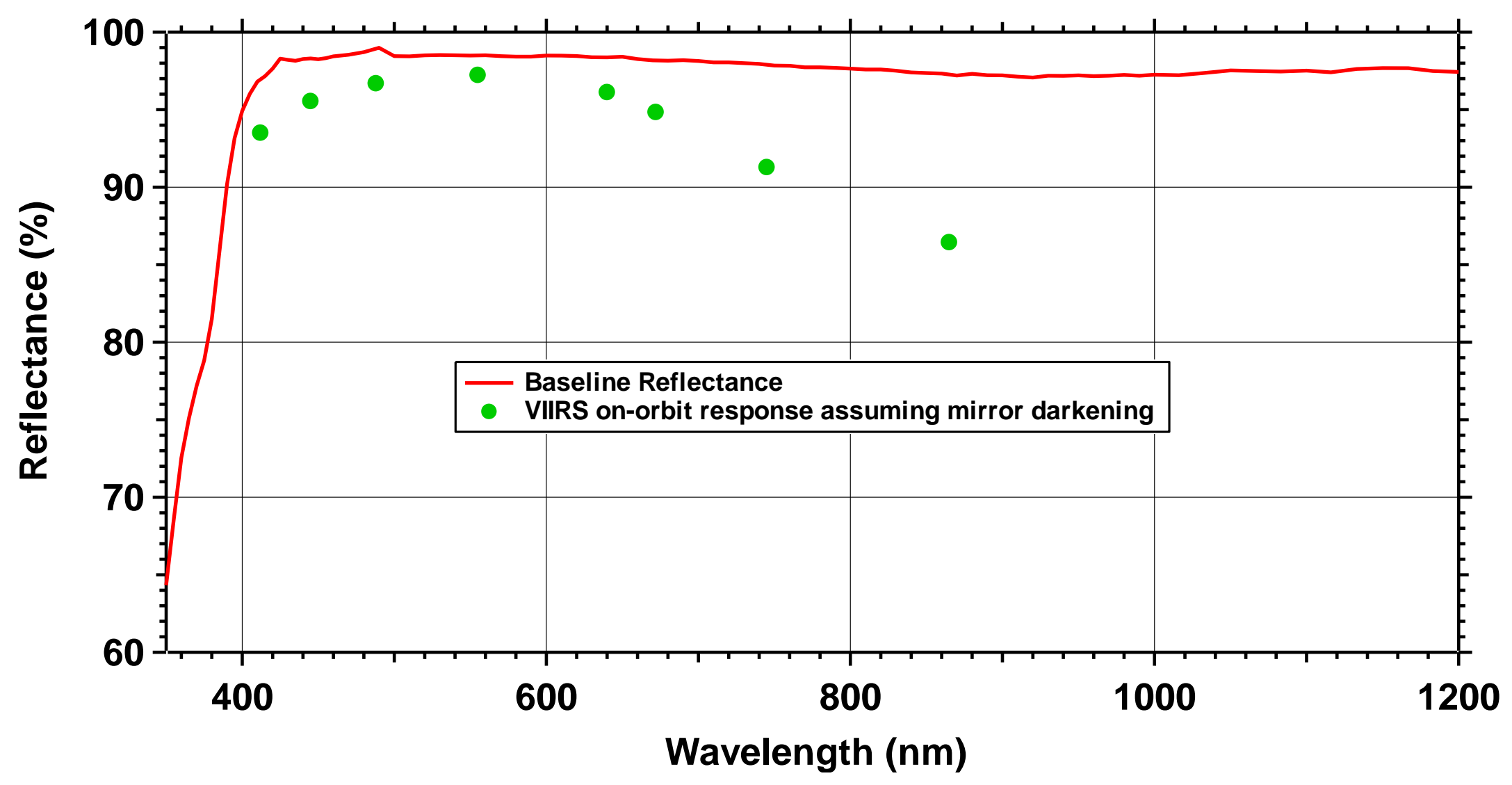

On-orbit response inferred from F-factor data (through orbit 882); assumes all observed degradation could be assigned to one mirror. 


\section{TWM Reflectance}

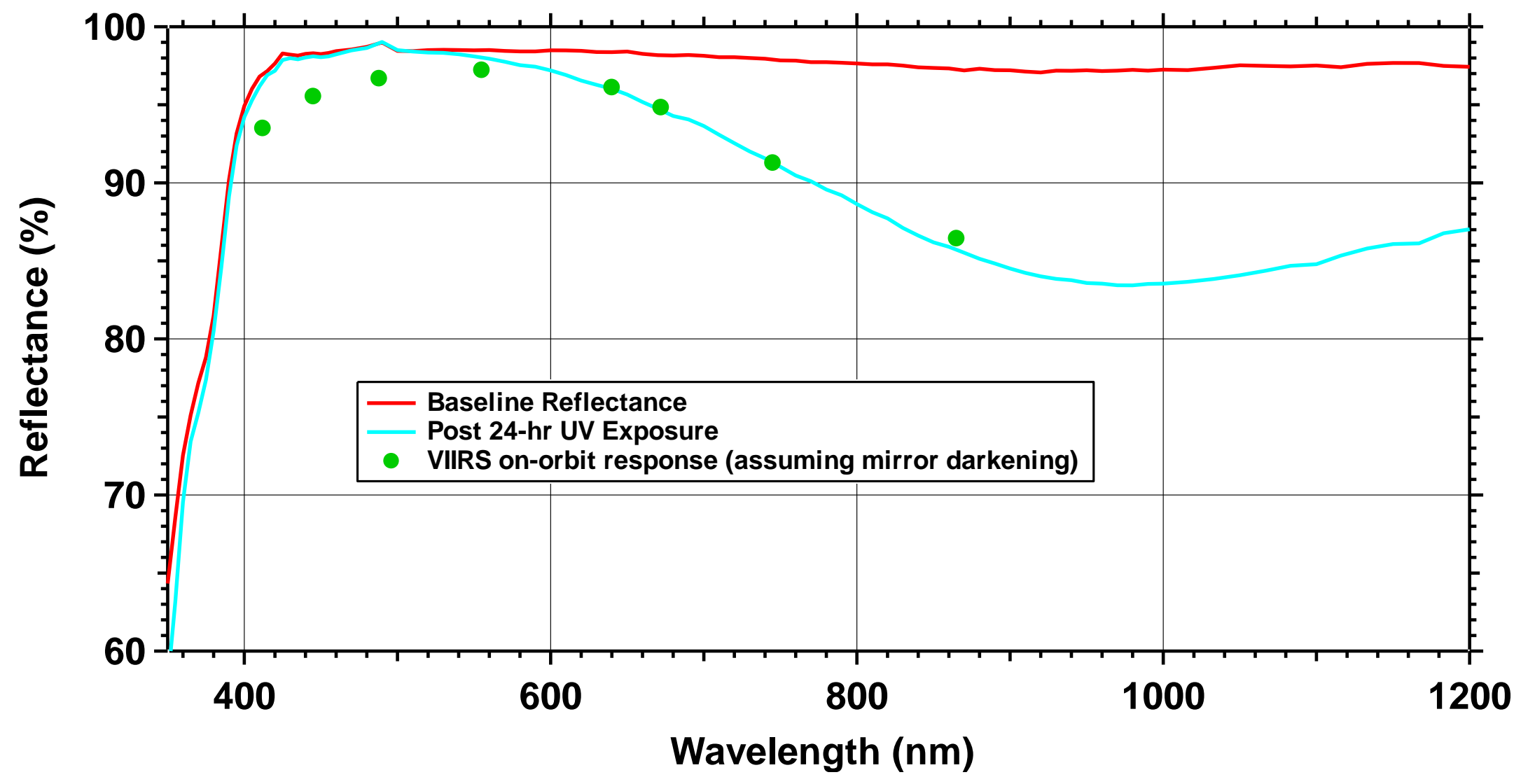

UV-induced degradation of TWM matches on-orbit VIIRS response, particularly for $\lambda>600 \mathrm{~nm}$. 


\section{TWM Reflectance}

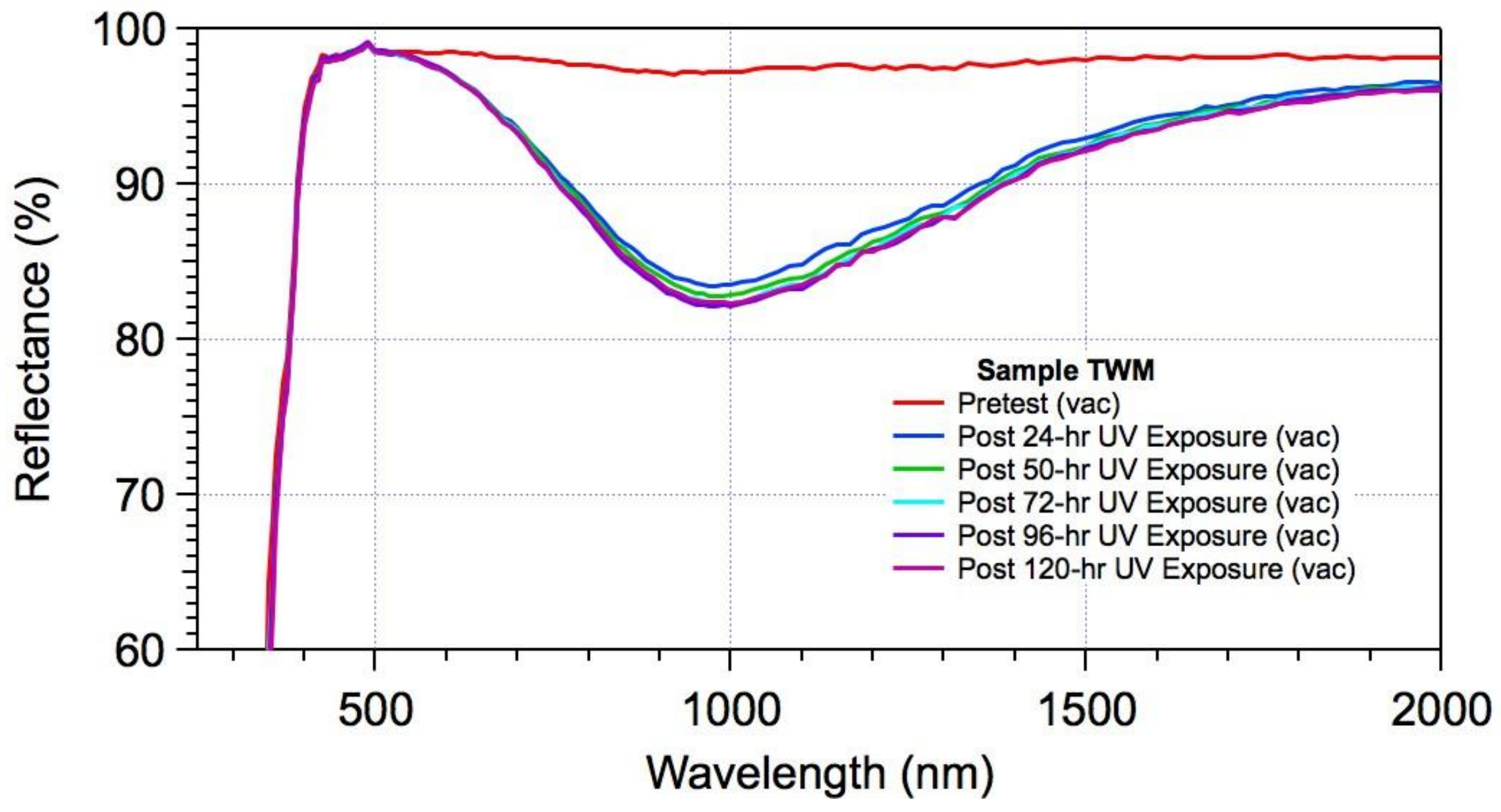

UV-induced degradation of TWM saturates after $\sim 3$ days of simulated exposure. 


\section{A3-31 Reflectance}

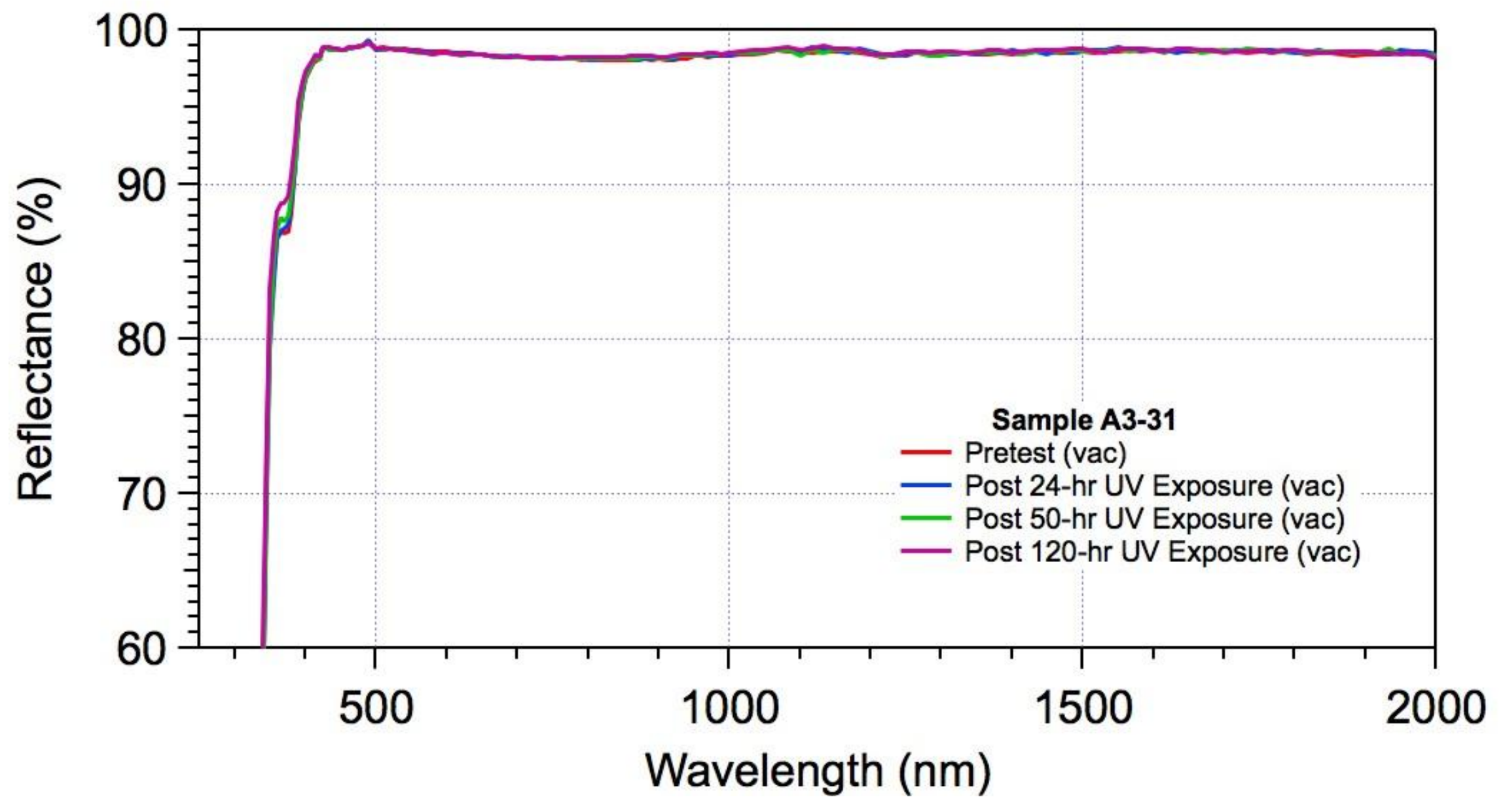

Mirror of the same design as TWM, but produced in a different coating run, was unaffected by the UV exposure. 


\section{Does the degradation anneal in vacuum?}

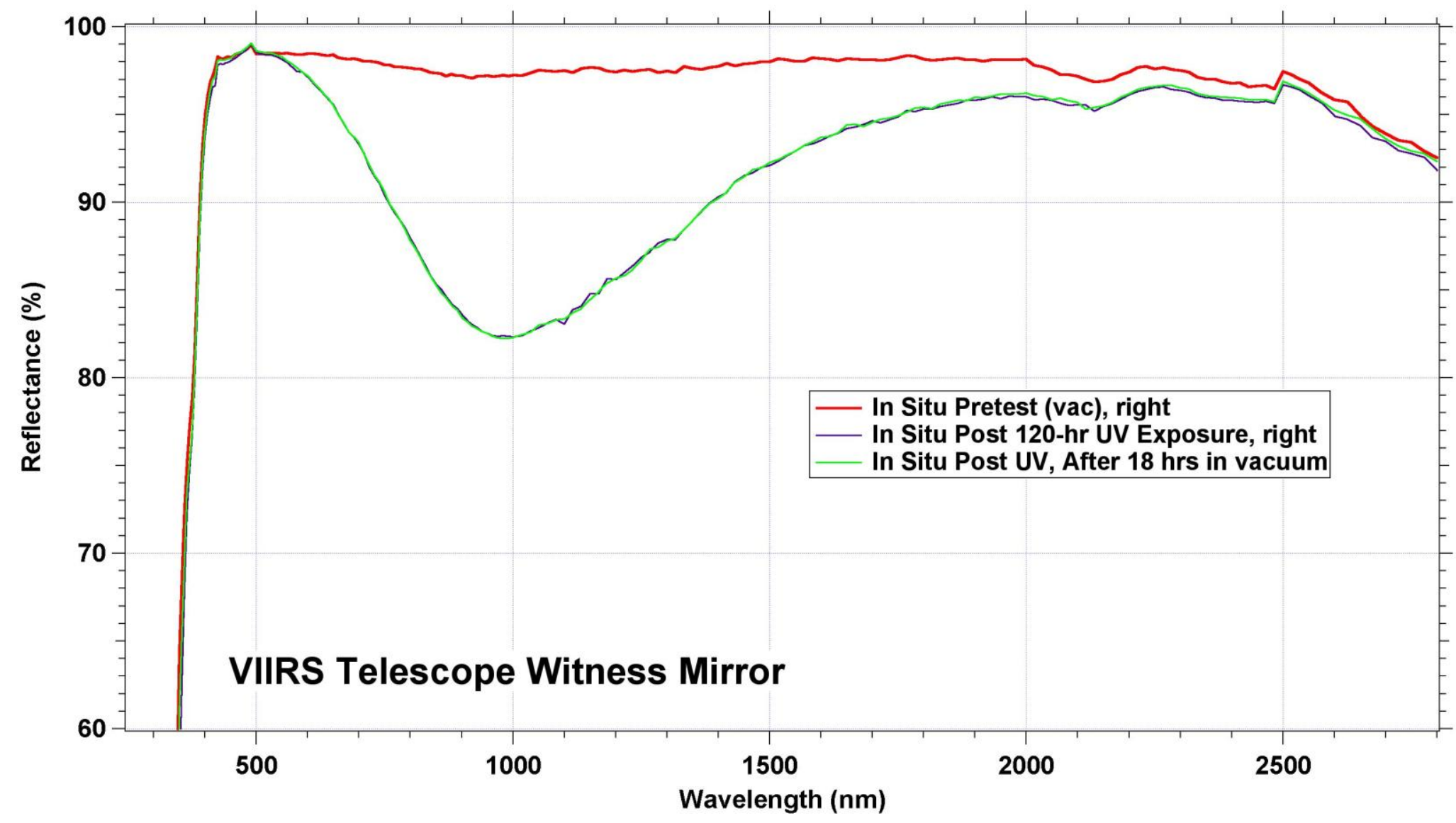

The reflectance loss persisted after the UV exposure ended. No change was observed overnight in vacuum at room temperature. 


\section{Does it recover upon exposure to air?}

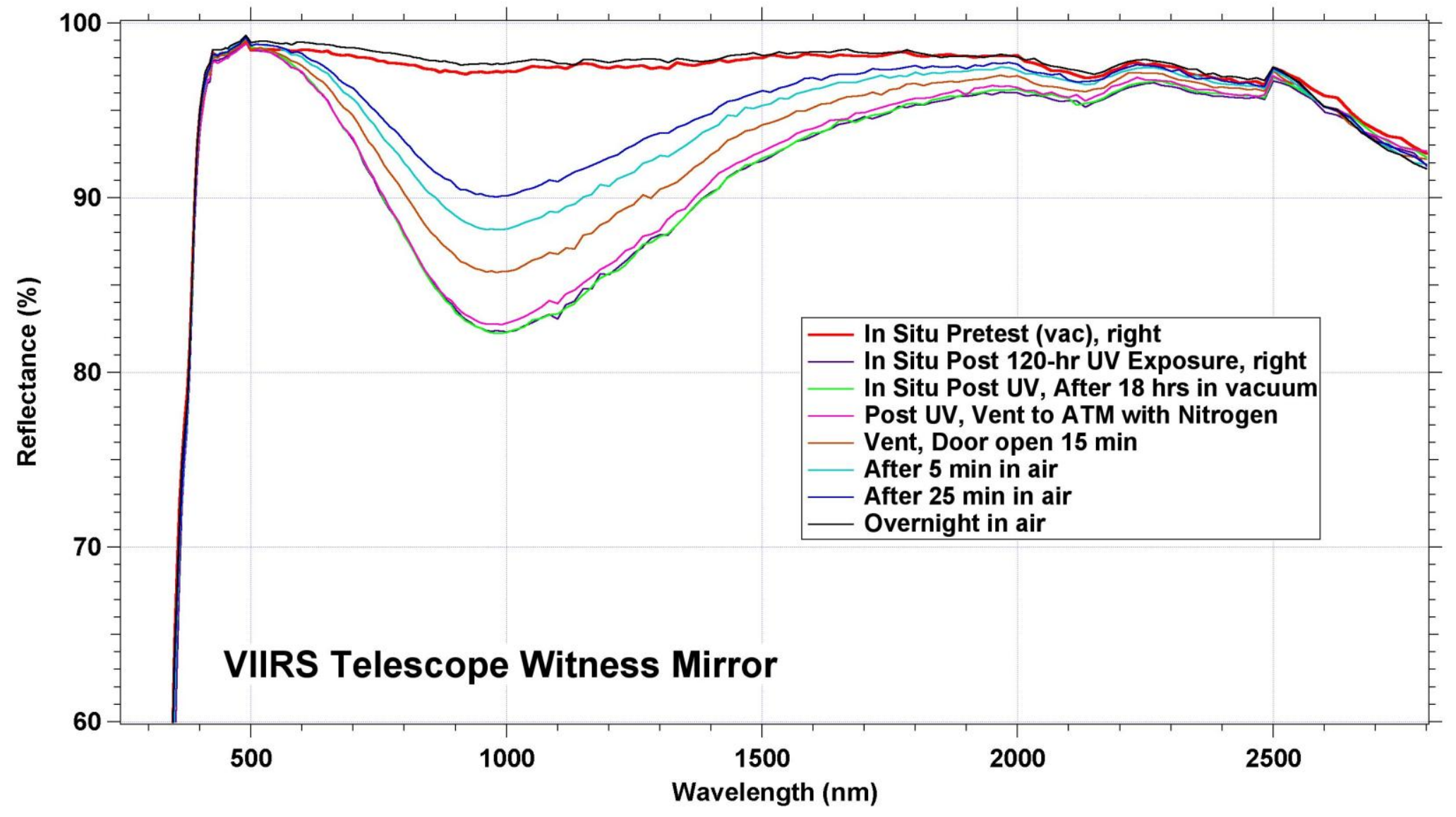

By the following morning, the degraded reflectance had bleached back to its pretest response. 


\section{Root-Cause Investigation Continues}

- Additional witness coupons of VIIRS mirrors arrive at The Aerospace Corporation

- A second witness mirror from the VIIRS RTA coating run (was bagged and stored after deposition - considered 'pristine') [designated CFM1]

- A witness mirror from the FMA coating run [designated FE94]

- Witness mirrors from other coating runs

- ToF-SIMS analysis performed

- Tungsten oxide also found on CFM1

- Tungsten oxide not detected on other mirrors

- Depth profiling provides further details about the mirrors

- Additional tungsten oxide under the mirror coating (at the interface with the substrate)

- A second UV exposure test performed 


\section{ToF-SIMS Depth Profile}

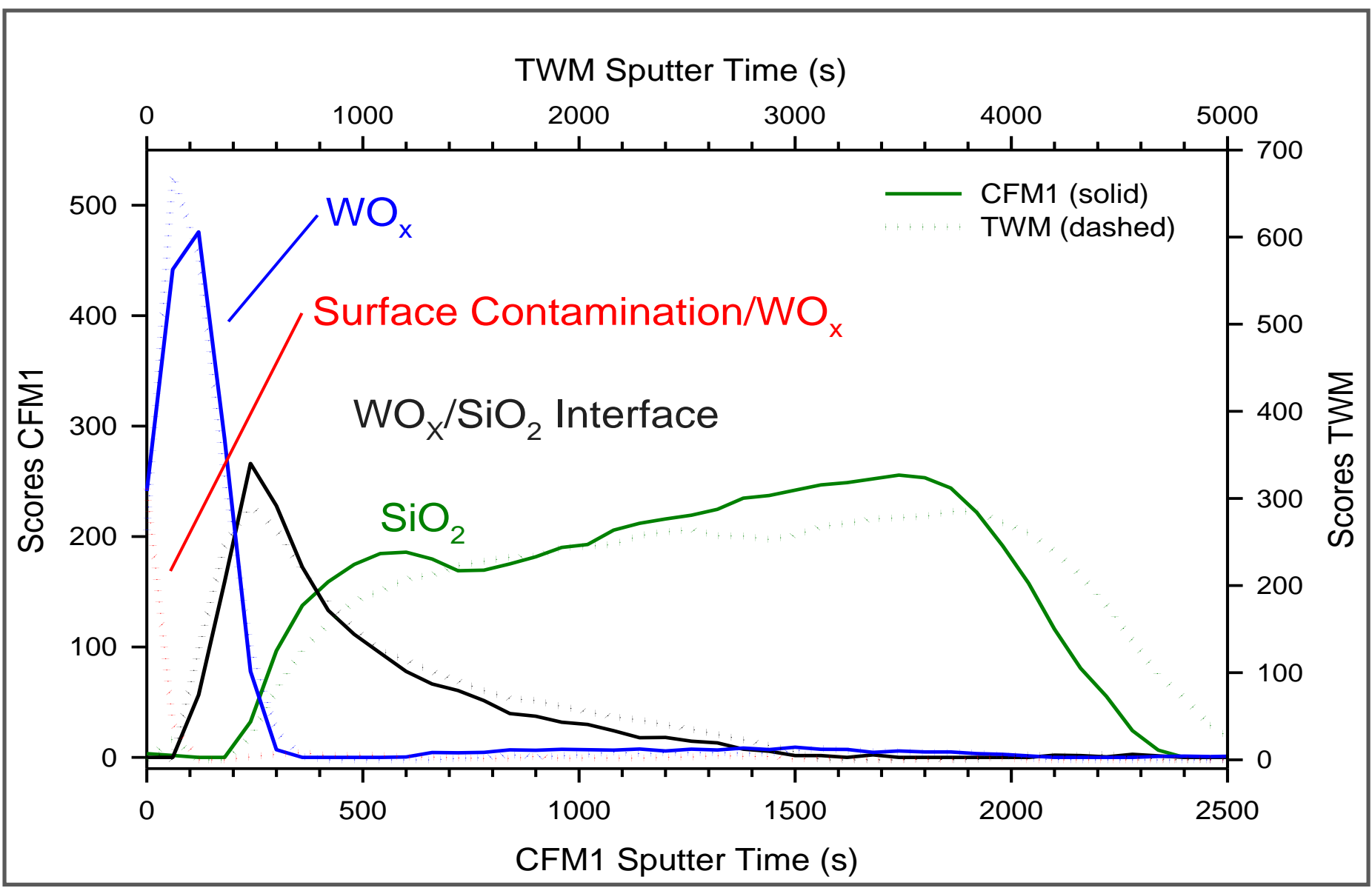

The two witness mirrors [from the RTA coating run] are very similar in composition; the surface of TWM shows contamination consistent with Al\&T. 


\section{ToF-SIMS Depth Profile}

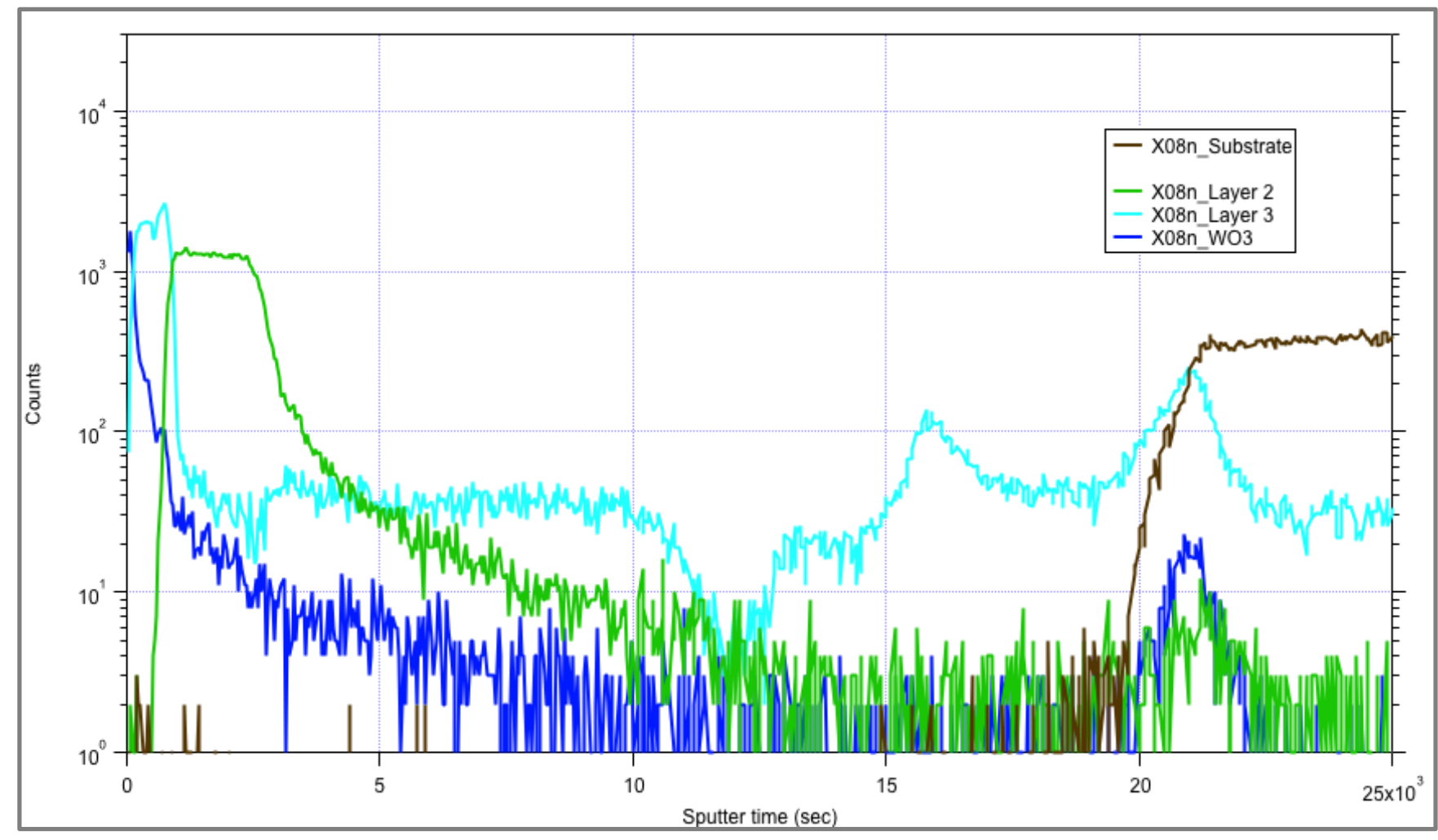

Tungsten oxide found in two places: A thin layer at the top of the coating and in smaller "trace" quantities below the silver layer. 


\section{TWM Reflectance - UV Test 2}

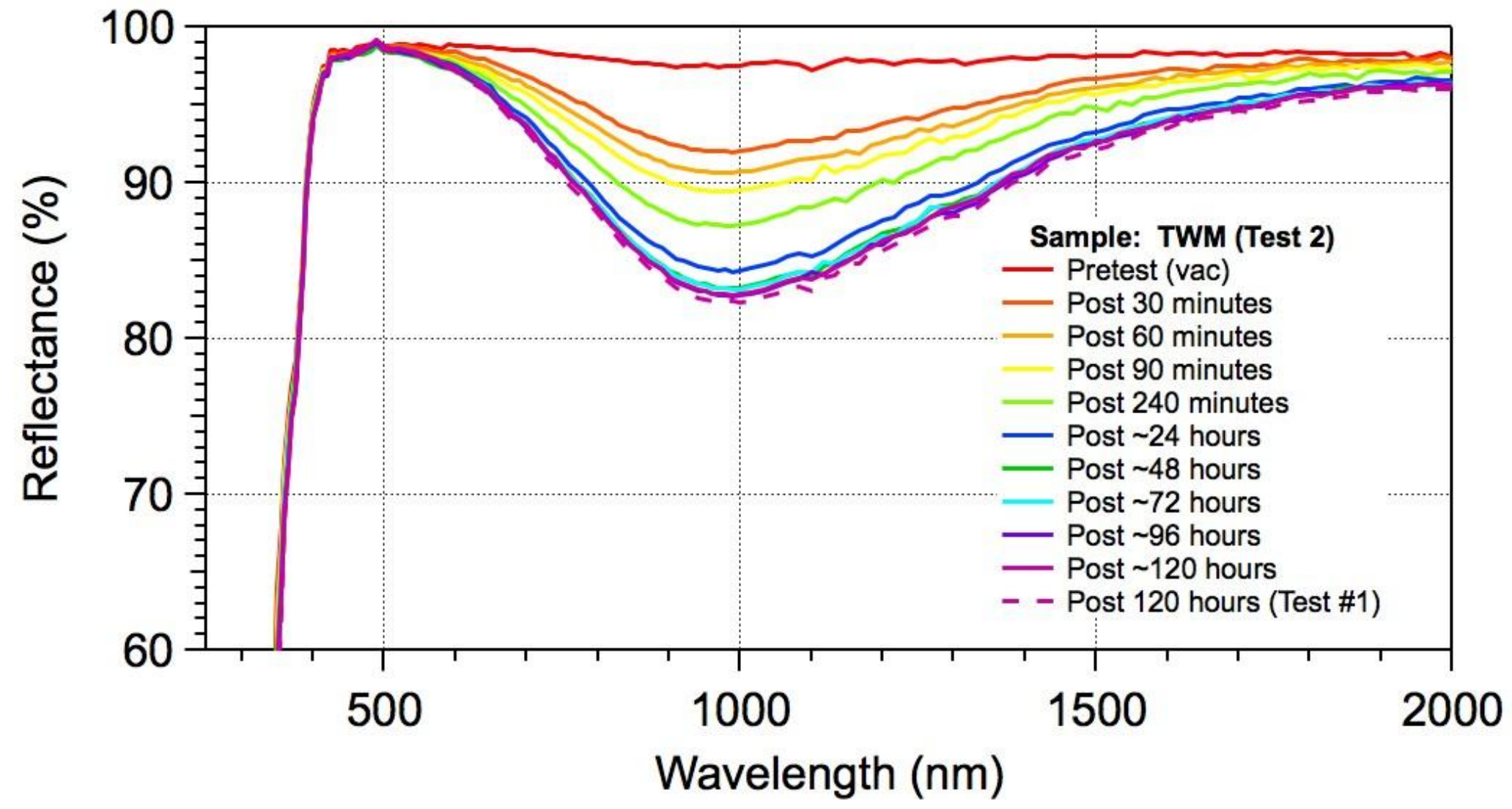

A second UV exposure induces reflectance loss similar to that in Test 1. 


\section{CFM1 Reflectance - UV Test 2}

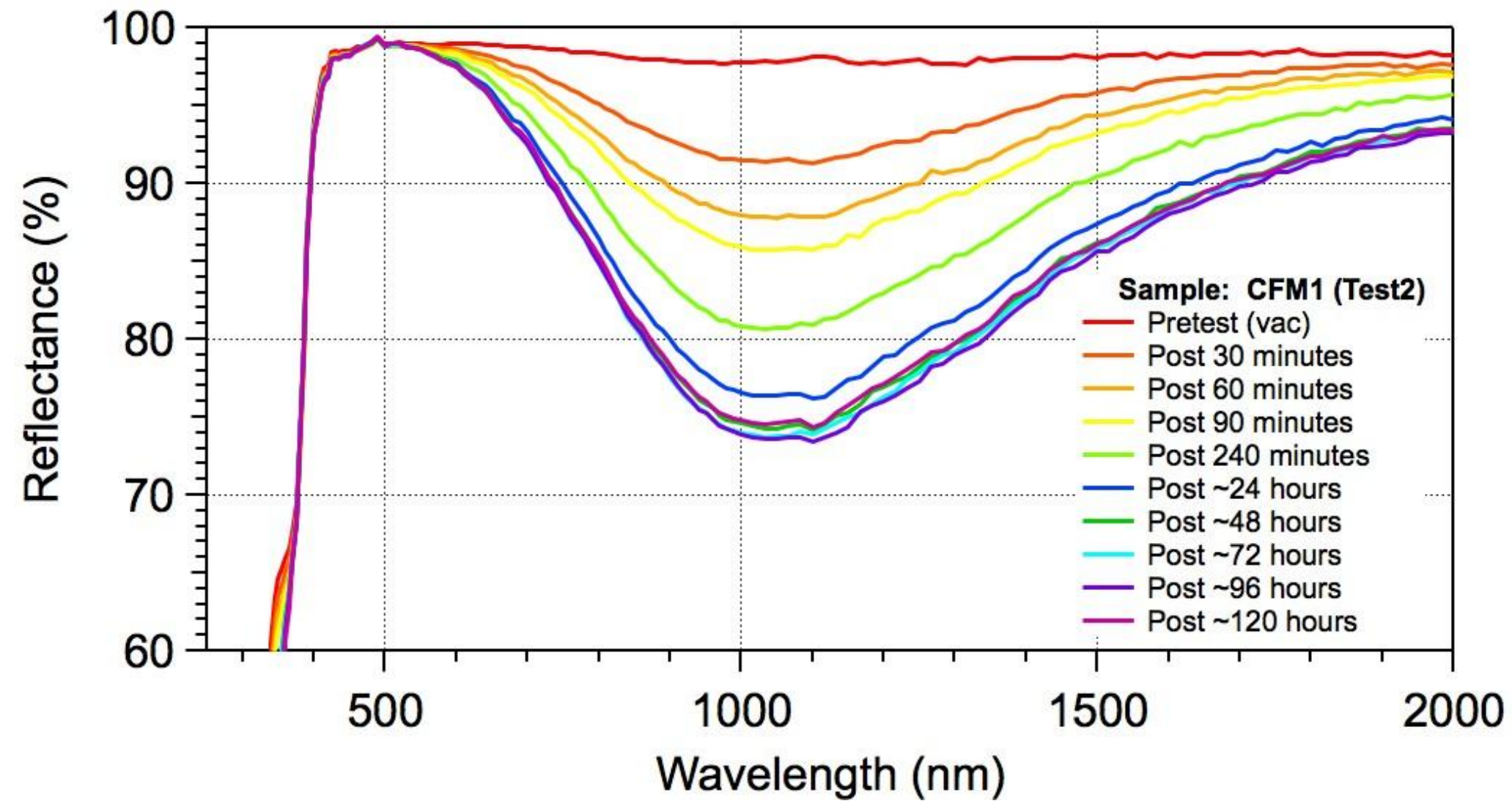

The second 'pristine' mirror [from the same coating run as the VIIRS RTA mirrors] is also degraded by UV exposure, with an even greater loss in reflectivity. This mirror was not exposed to Al\&T. 


\section{FE94 Reflectance - UV Test 2}

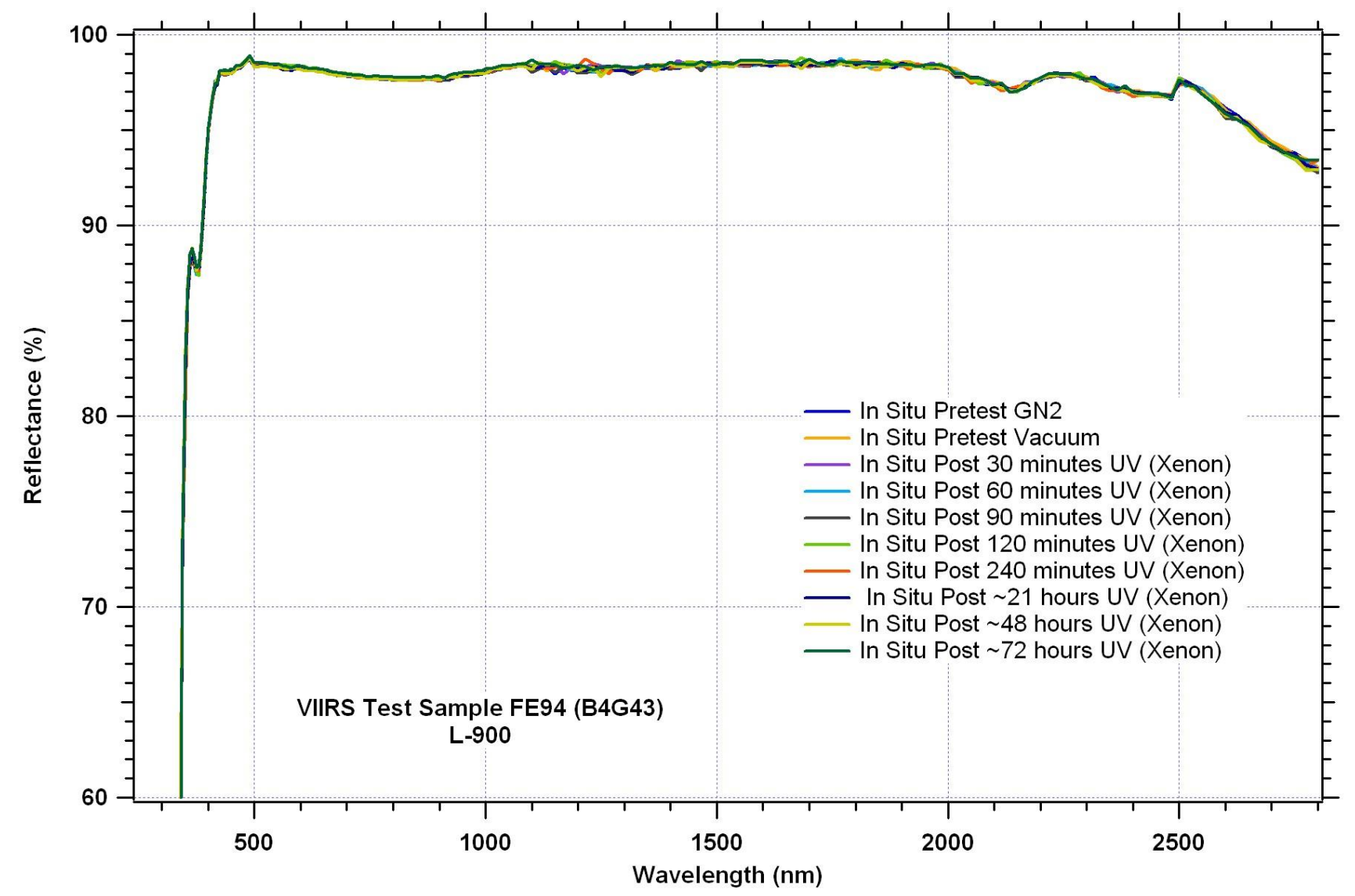

The mirror from a different coating run was unaffected by UV exposure. 


\section{Root-Cause}

- UV-induced degradation of tungsten oxide contaminated witness mirrors (TWM, CFM1) from RTA coating run

- The non-response of uncontaminated witness mirrors from other coating runs

\section{VIIRS on-orbit degradation likely due to} UV-induced darkening of RTA mirrors 


\section{Impact on VIIRS}

- All VIIRS telescope mirrors were coated/contaminated at the same time.

- The damage does not recover in vacuum.

- The Suomi orbit is too high for atomic oxygen to assist recovery; and the defects reappear with further UV exposure.

+ Only the primary mirror is exposed to the full intensity of Earth's albedo

+ Subsequent mirrors see diminished UV exposure, slowing their degradation. 


\section{RTA Coating History}

- After discovery of tungsten oxide on the surface of TWM, the vendor's coating records were reviewed.

- Vendor explained that the coating process includes cleaning substrates (prior to deposition) using an oxygen ion source.

- Oxygen ion source possesses Tungsten neutralizer filaments

- Explains tungsten oxide at coating/substrate interface

- The ion source remains off during the coating process

- RTA mirrors initially exhibited low reflectance, thought to be due to a lack of oxygen in the top dielectric layer of coating.

- The delivery of these completed mirrors was already behind schedule... 


\section{The Smoking Gun}

The vendor opted against stripping/recoating

- Proposed using the oxygen ion source to further oxidize top-coating

- Unqualified process - tested once on a single witness sample

- Not discussed with program's subjectmatter experts

- Process was hastily implemented (on a Sunday)

- No further testing of witness samples was considered

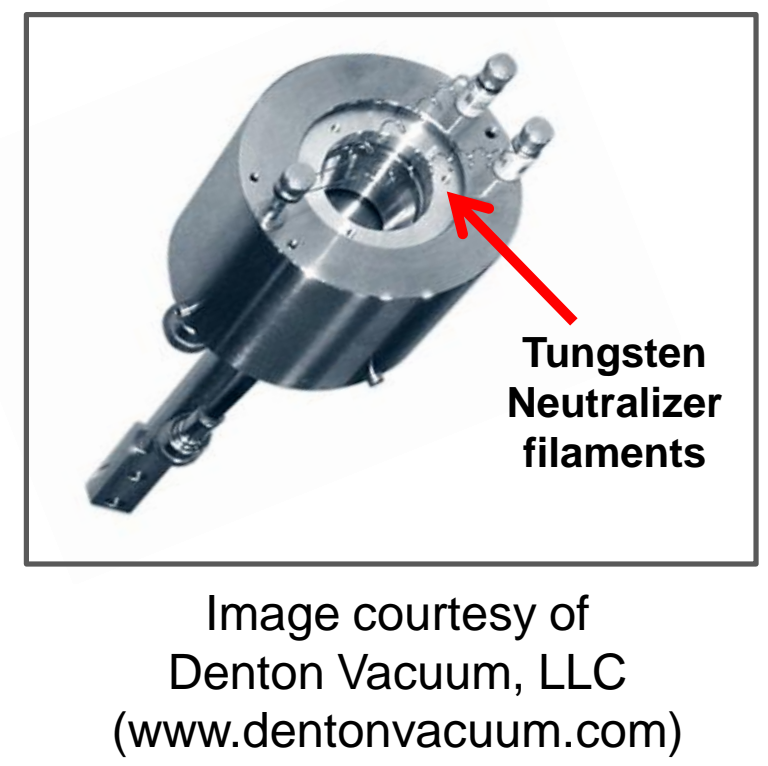




\section{Lessons Learned}

- Coating is still a "black art" - but we need to treat it like a science.

- Processes need to be fully qualified - and strictly followed

- Schedule pressures should not induce process deviations

- Changes need to be discussed with all stakeholders

- Beware unintended consequences of creative solutions

- Even good ideas need to be tested and verified

- Test as you fly (and fly as you test) - ensure that test specimens are fully representative of the flight article.

Ground-based testing remains a key facet of mission assurance 
Except where indicated, all photographs are the property of The Aerospace Corporation.

All trademarks, service marks, and trade names are the property of their respective owners. 


\section{Backups}




\section{Solar Simulation}

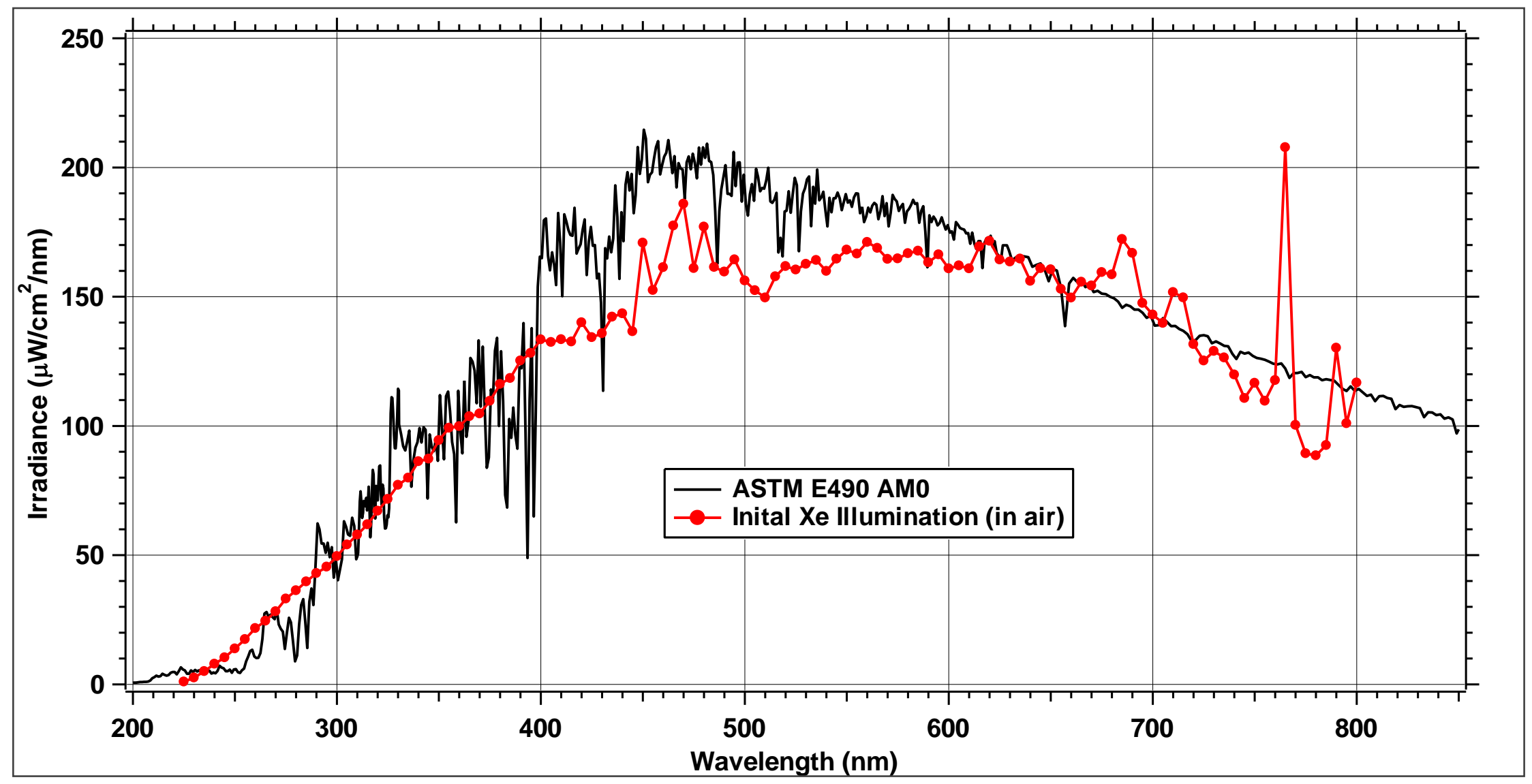

Radiometer measurement made prior to testing. 


\section{Albedo}

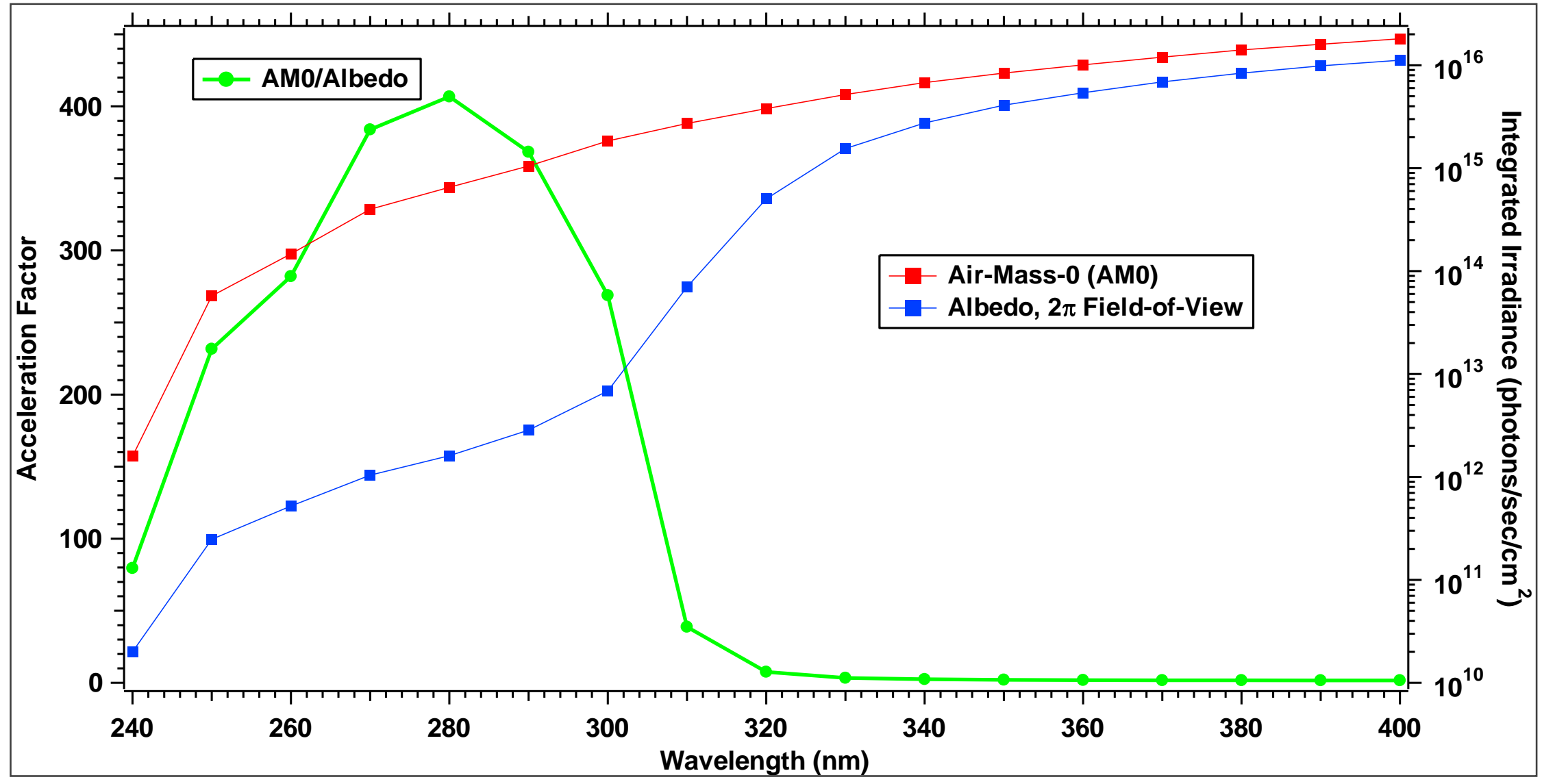

Albedo UV is different from direct solar exposure. 


\section{CERES Mirror - UV Test 1}

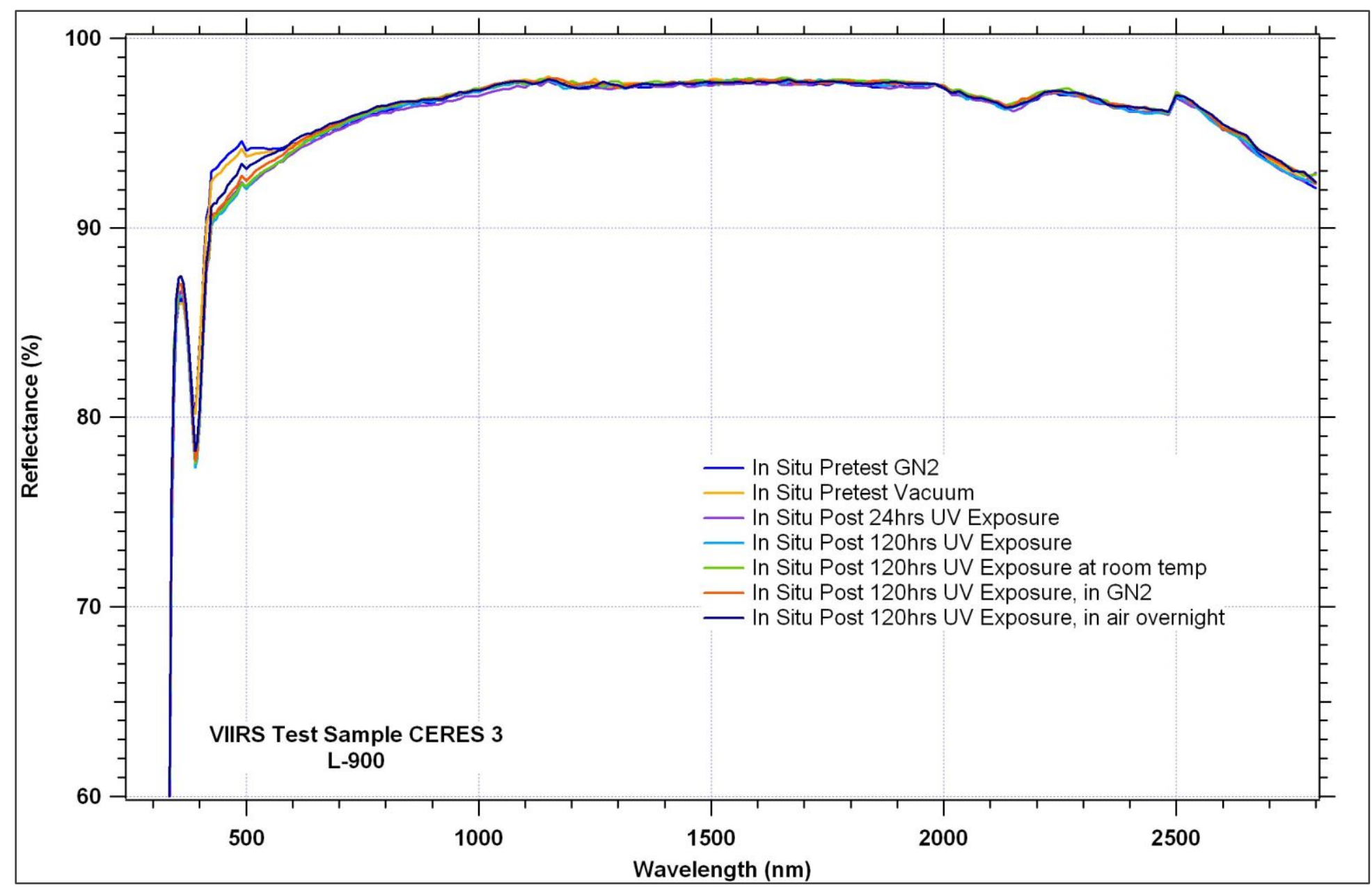

A CERES witness mirror, with a different spectrum than the VIIRS mirrors, was also exposed to UV during the test. The NIR absorption loss was not observed, but there was a slight decrease in short wavelength reflectance. 


\section{How Much Will NPP VIIRS Response Degrade?}

Aerospace trends VIIRS response and rate of response change twice per day in all VisNIR bands Longer wavelength bands are most affected $(\mathrm{I} 2 / \mathrm{M} 7=865 \mathrm{~nm}, \mathrm{M} 6=746 \mathrm{~nm}, \mathrm{M} 5=672 \mathrm{~nm})$
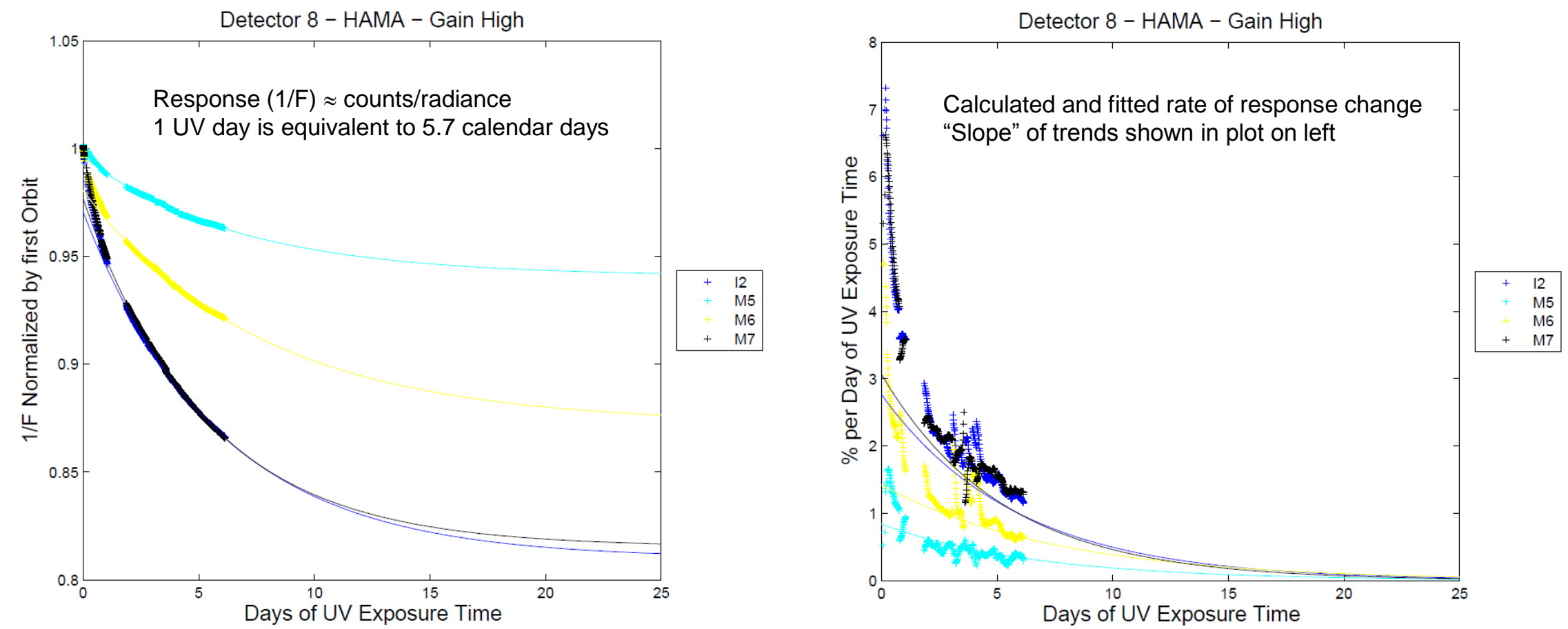

Rate of response change is slowing down as expected based saturation of darkening effect observed in witness sample measurements

\begin{tabular}{|l|c|c|c|c|}
\hline & $12-865 \mathrm{~nm}$ & $M 7-865 \mathrm{~nm}$ & M6 - 746 nm & M5 - 672 nm \\
\hline Extrapolated Max Response Loss & $19 \%$ & $18 \%$ & $13 \%$ & $6 \%$ \\
\hline SNR Margin Against Spec & $106 \%$ & $151 \%$ & $99 \%$ & $40 \%$ \\
\hline
\end{tabular}




\section{Continued loss from indirect UV}

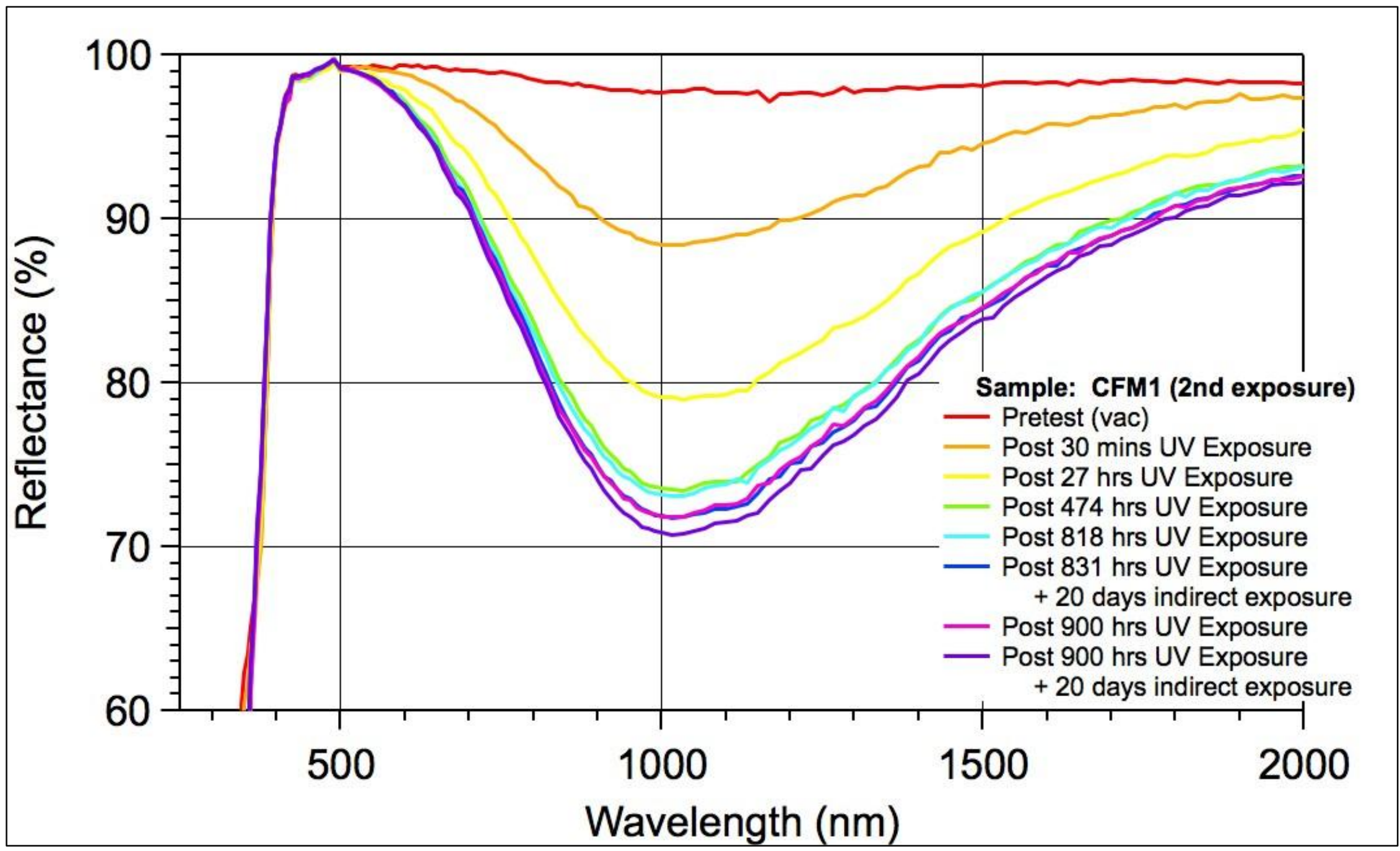




\section{Models help explain observed NIR losses}

What's going on? The protective layers on silver mirrors use interference to enhance the blue reflection from the mirror. We can model the electric field intensity within each layer.

- The $Y$ axis is the E-field squared

- $M$ is the incident medium, air

- 43 is the thin contamination layer and the high index layer

- 2 is the low index layer

- 1 is the silver layer, note the silver attenuates the field

- $S$ is the substrate, no light reaches the substrate.

- This pattern depends on the wavelength of incident light. For 630nm, the peak of the EFI is just below the interface between the high and low index layers.

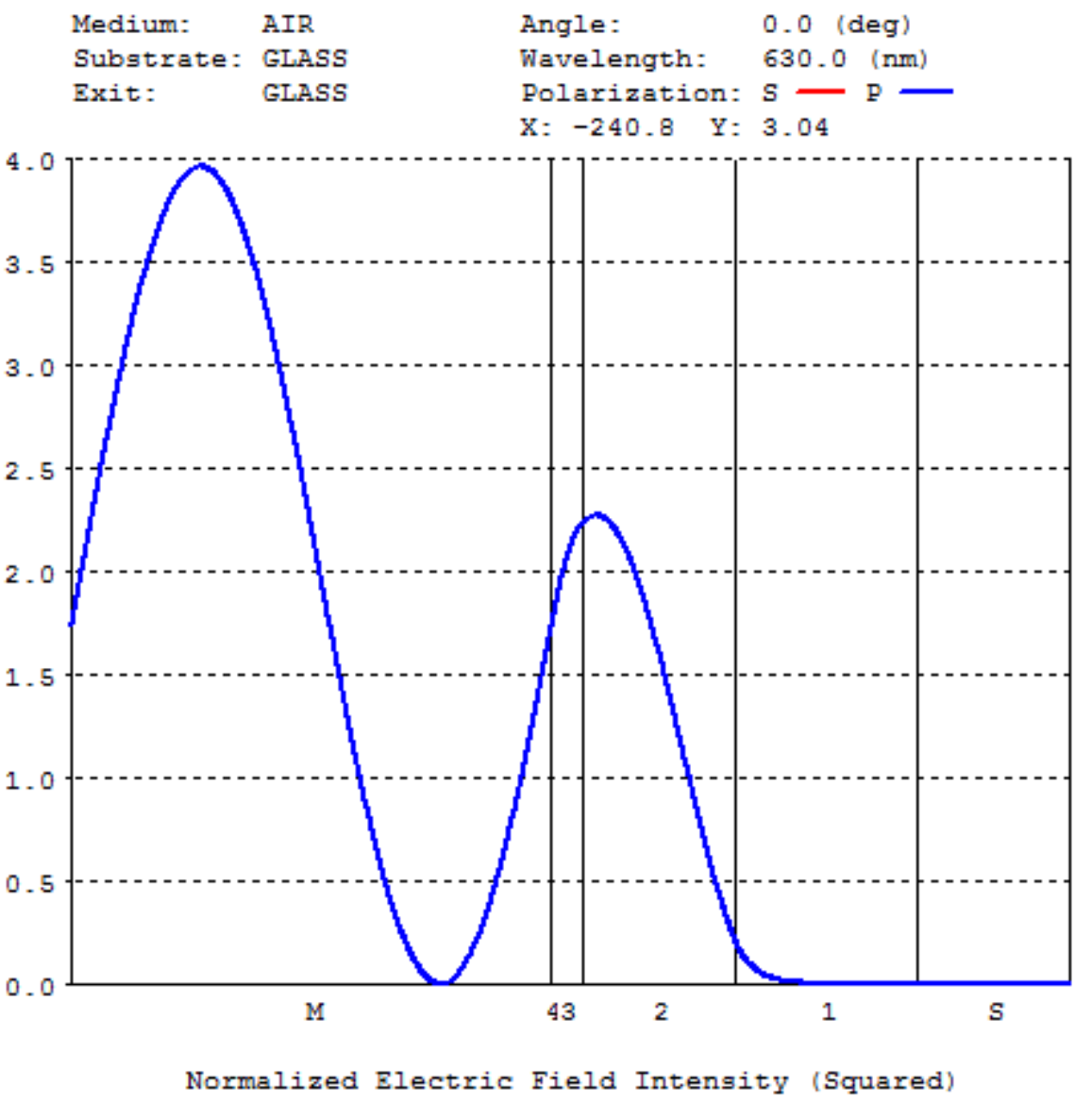

- There can be no absorption where there is no electric field! 
The electric field intensity at the top dielectric coating is peaked at $\sim 900 \mathrm{~nm}$ and is a node around $380 \mathrm{~nm}$. Thus at $380 \mathrm{~nm}$, there is no field to excite the absorber allowing it to absorb energy. At $900 \mathrm{~nm}$, the $\mathrm{E}$ field is maximized and therefore the energy loss is maximized.

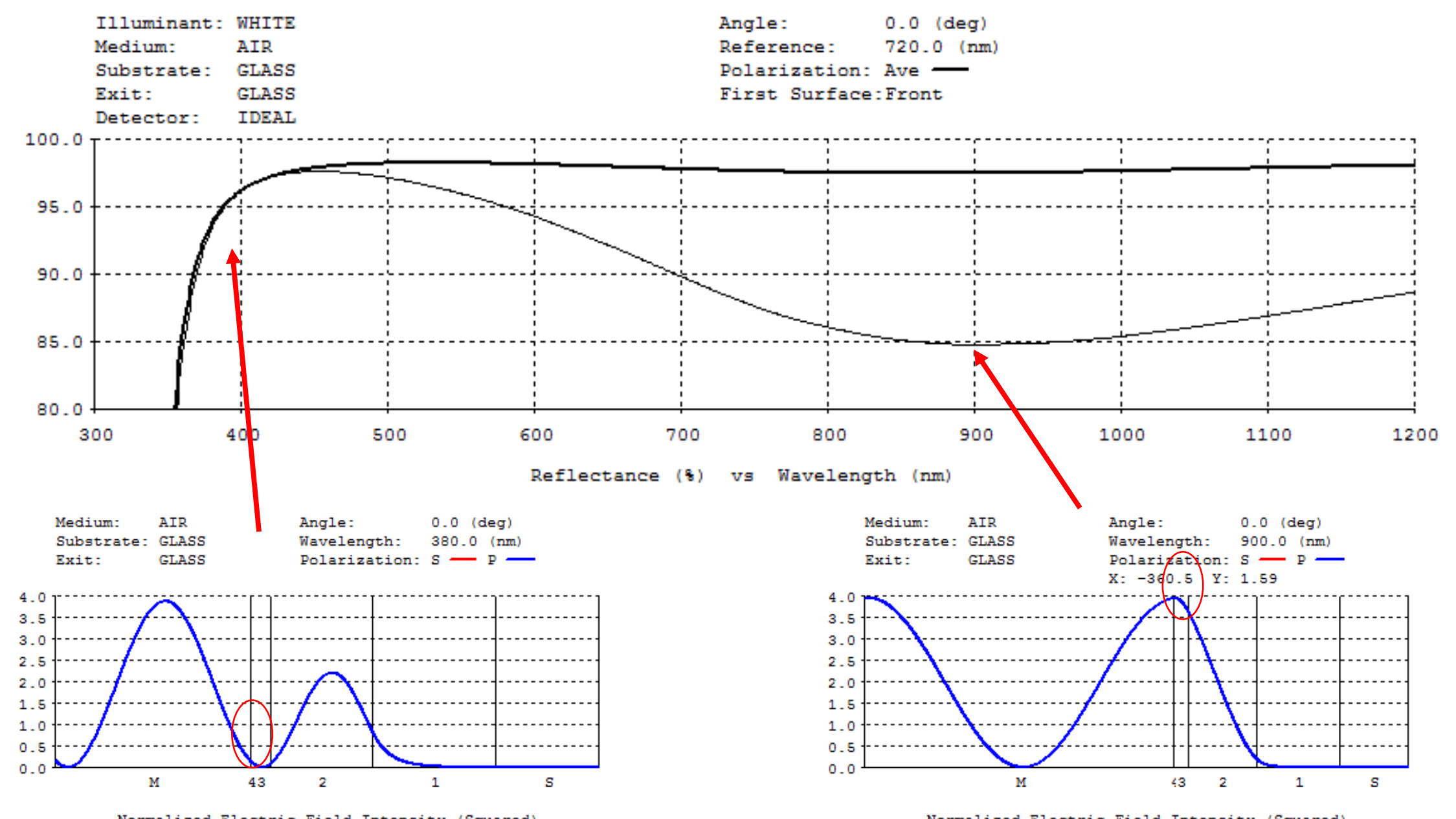

Normalized Electric Field Intensity (Squared)

Normalized Electric Field Intengity (Squared) 